\title{
A generalization of the topological Brauer group
}

\author{
A.V. Ershov
}

\begin{abstract}
In the present paper we study some homotopy invariants which can be defined by means of bundles with fiber a matrix algebra. In particular, we introduce some generalization of the Brauer group in the topological context and show that any its element can be represented as a locally trivial bundle with the structure group $\mathrm{N}_{k}^{\times}, k \in \mathbb{N}$. Finally, we discuss its possible applications in the twisted $K$-theory.
\end{abstract}

\section{Contents}

1 Matrix Grassmannians $\quad 3$

1.1 Basic definition . . . . . . . . . . . . . . . . . 3

1.2 Floating algebra bundles . . . . . . . . . . . . . . . . . . 4

1.3 The homogeneous space $\operatorname{Hom}\left(M_{k}(\mathbb{C}), M_{k l}(\mathbb{C})\right) \ldots \ldots$

1.4 The $\operatorname{Hom}\left(M_{k}(\mathbb{C}) . M_{k l}(\mathbb{C})\right)$-bundle $\mathbf{H}_{k, l}\left(A_{l^{u}}^{\text {univ }}\right) \stackrel{p}{\rightarrow} \operatorname{BPU}(k) \ldots$. . . . . . 8

2 The functor represented by the $H$-space $\mathrm{Gr} \quad 10$

2.1 The stable equivalence of FABs . . . . . . . . . . . . . . . . 10

2.2 The group structure . . . . . . . . . . . . . . . . . . 12

2.3 Some properties of FAB's core . . . . . . . . . . . . . . . . . . . . 12

2.4 Localization ........................ . . . . 13

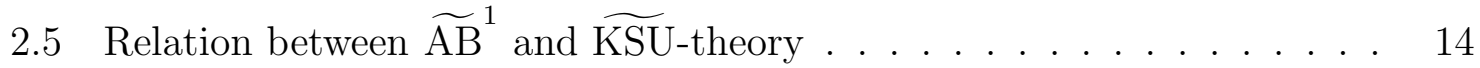

2.6 A U-version . . . . . . . . . . . . . . . . . . . . . 15

3 Homotopy invariants related to algebra bundles $\quad 16$

3.1 Reminder: The classical Brauer group . . . . . . . . . . . . . . 16

3.2 An obstruction for an embedding of a locally trivial algebra bundle into a trivial ond 19

3.3 A generalized Brauer group . . . . . . . . . . . . . . . . . . . 22

3.4 Application: the twisted $K$-theory . . . . . . . . . . . . . . . . . . . . . 32

\section{Introduction}

The aim of this paper is to study different homotopy invariants of $C W$-complexes which can be constructed by means of locally-trivial bundles with fiber a matrix algebra. An 
example of such invariant is the first obstruction for an embedding of a locally-trivial matrix algebra bundle into a trivial one (under some extra conditions which will be formulated below). Another invariant we shall deal with is a homotopy functor $\mathrm{GBr}$ which can be treated as a generalization of the classical "topological" Brauer group. So let us remember the basic idea of the Brauer group.

Suppose $X$ is a finite $C W$-complex. The topological Brauer group $\operatorname{Br}(X)$ can be defined as the group of equivalence classes of locally-trivial bundles $A_{k}$ over $X$ with fibers $M_{k}(\mathbb{C})$ (for arbitrary $k \in \mathbb{N}$ ) with respect to the following equivalence relation:

$$
A_{k} \sim B_{l} \Leftrightarrow A_{k} \otimes \operatorname{End}\left(\xi_{m}\right) \cong B_{l} \otimes \operatorname{End}\left(\eta_{n}\right)
$$

for some complex vector bundles $\xi_{m}, \eta_{n}$ of rank $m, n$ respectively (in particular, $k m=$ $\ln )$. Roughly speaking, we can say that $\operatorname{Br}(X)$ is the group of obstructions for the lifting of locally trivial bundles $A_{k}$ over $X$ with fiber the matrix algebra $M_{k}(\mathbb{C})$ to bundles of the form $\operatorname{End}\left(\xi_{k}\right)$ for some locally trivial $\mathbb{C}^{k}$-bundle $\xi_{k}$ over $X$.

In order to define the generalized Brauer group $\mathrm{GBr}$ for any natural $k$ we introduce some $C^{*}$-algebra $\mathrm{N}_{k} \subset M_{k}(\mathcal{B}(H)$ ), where $\mathcal{B}(H)$ is the algebra of bounded operators in a separable complex Hilbert space $H$, and consider bundles with the group of its invertible elements $\mathrm{N}_{k}^{\times}$as a structure group. Some of such bundles come from finite-dimensional vector bundles due to a group homomorphism $\mathrm{U}(k) \rightarrow \mathrm{N}_{k}^{\times}$. The generalized Brauer group is just the group of equivalence classes of $\mathrm{N}_{k}^{\times}$-bundles modulo those that come from finite-dimensional vector bundles.

According to a classical theorem of J.-P. Serre, there is an isomorphism $\operatorname{Br}(X) \cong$ $H_{\text {tors }}^{3}(X ; \mathbb{Z})[7$. In other words, all obstructions for the lifting of locally-trivial bundles $A_{k}$ over $X$ with fiber $M_{k}(\mathbb{C})$ to bundles of the form $\operatorname{End}\left(\xi_{k}\right)$ are independent of the higher-dimensional integer cohomology of dimensions greater than 3 . In contrast to the classical case, the generalized Brauer group $\operatorname{GBr}(X)$ actually depends on the higherdimensional cohomology $H_{\text {tors }}^{2 i+1}(X ; \mathbb{Z}), i \geq 2$. On the other hand, just as in the classical group $\operatorname{Br}(X)$, any element of $\operatorname{GBr}(X)$ has finite order.

The last part of the paper is motivated by the recent progress in the twisted $K$ theory. For any bundle with the structure group $\mathrm{N}_{k}^{\times}$we define the corresponding twisted $K$-group. We hope that our approach will provide an interesting example of more general twistings than the ones considered up to now.

Acknowledgments I am grateful to E.V. Troitsky for constant attention to this work and all-round support and to V.M. Manuilov and A.S. Mishchenko for very helpful discussions. 


\section{Matrix Grassmannians}

In this section we give definitions of objects we shall deal with below. More precisely, we start with so-called "matrix Grassmannians" (which play the same role for matrix algebras as the usual Grassmannians for vector spaces) and try to develop a theory of matrix algebra bundles by analogy with the classical theory of vector bundles. We restrict ourselves to considering a special class of matrix algebra bundles (so-called "floating" bundles), because only in this case we avoid the localization (hence a trivialization) of their theory. Finally in this way we obtain an invariant of $C W$-complexes closely connected with the usual $K$-theory (cf. Theorem 21).

Note that in the course of the paper the basic field is $\mathbb{C}$.

\subsection{Basic definition}

Let us remember that the Grassmannian $\mathrm{G}_{k, n}, 0 \leq k \leq n$ is a homogeneous space parameterizing $k$-dimensional subspaces in the fixed $n$-dimensional vector space $\mathbb{C}^{n}$.

By analogy with this definition we introduce a "matrix Grassmannian" which parameterizes central matrix subalgebras of a given dimension in the fixed matrix algebra.

First, remember that a central subalgebra is a subalgebra whose center coincides with the center of the whole algebra (i.e. with the field of scalar matrices in the case of matrix algebras). In particular, the identity matrix of the "big" algebra is the identity element of any central subalgebra. By $M_{n}(\mathbb{C})$ denote the algebra of all $n \times n$ matrices over $\mathbb{C}$.

A central subalgebra in $M_{n}(\mathbb{C})$ isomorphic to $M_{k}(\mathbb{C})$ is called a $k$-subalgebra, for short. Note that such a subalgebra exists only if $k \mid n$, i.e. $n=k l$ for some natural $l$.

Definition 1. The matrix Grassmannian $\mathrm{Gr}_{k, l}^{\prime}$ is a homogeneous space which parametrizes $k$-subalgebras in a fixed matrix algebra $M_{k l}(\mathbb{C})$.

Proposition 2. For any pair $k, l>1$ there exists the matrix Grassmannian $\mathrm{Gr}_{k, l}^{\prime}$.

Proof follows from Noether-Skolem's theorem [10], § 12.6.

Noether-Skolem's theorem also implies that $\mathrm{Gr}_{k, l}^{\prime}$ is a homogeneous space of the group $\mathrm{PGL}_{k l}(\mathbb{C})$ represented as follows:

$$
\mathrm{Gr}_{k, l}^{\prime}=\mathrm{PGL}_{k l}(\mathbb{C}) / \mathrm{PGL}_{k}(\mathbb{C}) \otimes \mathrm{PGL}_{l}(\mathbb{C}),
$$

where $\mathrm{PGL}_{k}(\mathbb{C}) \otimes \mathrm{PGL}_{l}(\mathbb{C})$ denotes the image of the embedding $\mathrm{PGL}_{k}(\mathbb{C}) \times \mathrm{PGL}_{l}(\mathbb{C}) \hookrightarrow$ $\mathrm{PGL}_{k l}(\mathbb{C})$ induced by the Kronecker product of matrices.

There is a tautological $M_{k}(\mathbb{C})$-bundle over the matrix Grassmannian $\mathrm{Gr}_{k, l}^{\prime}$ whose total space $\mathcal{A}_{k, l}^{\prime}$ is defined in the following way:

$$
\mathcal{A}_{k, l}^{\prime}:=\left\{(x, T) \mid x \in \mathrm{Gr}_{k, l}^{\prime}, T \in M_{k, x}\right\} \subset \mathrm{Gr}_{k, l}^{\prime} \times M_{k l}(\mathbb{C}),
$$


where $M_{k, x} \subset M_{k l}(\mathbb{C})$ denotes the $k$-subalgebra corresponding to a point $x \in \mathrm{Gr}_{k, l}^{\prime}$, and the projection is induced by the projection of the trivial bundle onto the first factor.

The noncompact space $\mathrm{Gr}_{k, l}^{\prime}$ can be replaced by a homotopy equivalent compact one $\mathrm{Gr}_{k, l}$. More precisely, consider the "standard" Hermitian metric $\langle A, B\rangle=\operatorname{tr}\left(A \bar{B}^{t}\right)$ on $M_{k l}(\mathbb{C})$. We say that a $k$-subalgebra $M_{k, x} \subset M_{k l}(\mathbb{C})$ is unitary if $M_{k, x}=$

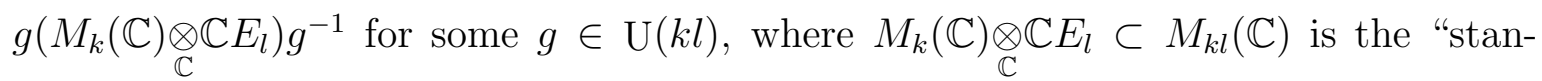
dard" $k$-subalgebra.

Let $\mathrm{PU}(n)$ be the projective unitary group, i.e. the quotient group $\mathrm{U}(n) /\left\{\exp (i \varphi) E_{n} \mid \varphi \in \mathbb{R}\right\}$. Put

$$
\mathrm{Gr}_{k, l}:=\mathrm{PU}(k l) / \mathrm{PU}(k) \otimes \mathrm{PU}(l)
$$

Obviously, it is a subspace in $\mathrm{Gr}_{k, l}^{\prime}$ which parameterizes unitary $k$-subalgebras.

In just the same way as in the noncompact case, one can define the tautological $M_{k}(\mathbb{C})$-bundle $\mathcal{A}_{k, l} \subset \mathrm{Gr}_{k, l} \times M_{k l}(\mathbb{C})$ over $\mathrm{Gr}_{k, l}$.

Recall that the group $\mathrm{U}(n)(\mathrm{PU}(n))$ is a strong deformation retract of $\mathrm{GL}_{n}(\mathbb{C})$ $\left(\mathrm{PGL}_{n}(\mathbb{C})\right.$ respectively). Hence there is a homotopy equivalence $\mathrm{Gr}_{k, l} \simeq \mathrm{Gr}_{k, l}^{\prime}$. Because of this equivalence, we shall not distinguish these spaces below.

\section{$1.2 \quad$ Floating algebra bundles}

The classical Grassmannians are classifying spaces for vector bundles. In this subsection we introduce a class of bundles that are classified by matrix Grassmannians $\mathrm{Gr}_{k, l}$ (under the extra condition $(k, l)=1$ whose sense will be clarified below).

Let $X$ be a finite $C W$-complex. By $\widetilde{M}_{n}$ denote a trivial bundle (over $X$ ) with fiber $M_{n}(\mathbb{C})$ (note that in general a trivialization on $\widetilde{M}_{n}$ is not supposed to be given).

Definition 3. Let $A_{k}(k>1)$ be a locally trivial bundle over $X$ with fiber $M_{k}(\mathbb{C})$. Assume that there is a bundle map $\mu$

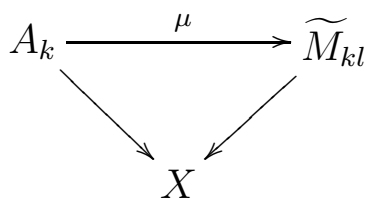

such that for any point $x \in X$ it embeds the fiber $\left(A_{k}\right)_{x} \cong M_{k}(\mathbb{C})$ into the fiber $\left(\widetilde{M}_{k l}\right)_{x} \cong M_{k l}(\mathbb{C})$ as a central simple subalgebra, and the positive integers $k, l$ are relatively prime (i.e. their greatest common divisor $(k, l)=1$ ). Then the triple $\left(A_{k}, \mu, \widetilde{M}_{k l}\right)$ is called a floating algebra bundle (abbrev. FAB) over $X$. The locally trivial bundle $A_{k}$ is called a core of the $\operatorname{FAB}\left(A_{k}, \mu, \widetilde{M}_{k l}\right)$. 
Remark 4. Let $A$ be a central simple algebra over a field $\mathbb{K}, B \subset A$ a central simple subalgebra in $A$. It is well known that the centralizer $\mathrm{Z}_{A}(B)$ of $B$ in $A$ is a central simple subalgebra in $A$ again, moreover, the equality $A=B \underset{\mathbb{K}}{\otimes} \mathrm{Z}_{A}(B)$ holds [10], $\S 12.7$. Taking centralizers for all fibers of the subbundle $A_{k} \subset \widetilde{M}_{k l}$ in the corresponding fibers of the trivial bundle $\widetilde{M}_{k l}$, we get the complementary subbundle $B_{l}$ with fiber $M_{l}(\mathbb{C})$ together with its embedding $\nu: B_{l} \hookrightarrow \widetilde{M}_{k l}$. Moreover, $A_{k} \otimes B_{l}=\widetilde{M}_{k l}$.

Conversely, to a given pair $\left(A_{k}, B_{l}\right)$ consisting of $M_{k}(\mathbb{C})$-bundle $A_{k}$ and $M_{l}(\mathbb{C})$ bundle $B_{l}$ over $X$ such that $A_{k} \otimes B_{l}=\widetilde{M}_{k l}$, we can construct a unique triple $\left(A_{k}, \mu, \widetilde{M}_{k l}\right)$, where $\mu$ is the embedding $A_{k} \hookrightarrow A_{k} \otimes B_{l}, a \mapsto a \otimes 1_{B_{l}}$.

Definition 5. A morphism from a FAB $\left(A_{k}, \mu, \widetilde{M}_{k l}\right)$ to a $F A B\left(C_{m}, \nu, \widetilde{M}_{m n}\right)$ over $X$ is a pair $(f, g)$ of bundle maps $f: A_{k} \hookrightarrow C_{m}, g: \widetilde{M}_{k l} \hookrightarrow \widetilde{M}_{m n}$ such that

- $f, g$ are fiberwise homomorphisms of central algebras (i.e. they actually are embeddings);

- the square diagram

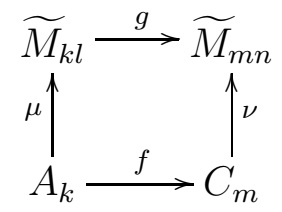

commutes;

- let $B_{l} \subset \widetilde{M}_{k l}, D_{n} \subset \widetilde{M}_{m n}$ be the complementary subbundles for $A_{k}, C_{m}$, respectively (see the remark above), then $g$ maps $B_{l}$ into $D_{n}$.

Note that a morphism $(f, g):\left(A_{k}, \mu, \widetilde{M}_{k l}\right) \rightarrow\left(C_{m}, \nu, \widetilde{M}_{m n}\right)$ exists only if $k|m, l| n$.

In particular, an isomorphism between FABs $\left(A_{k}, \mu, \widetilde{M}_{k l}\right)$ and $\left(C_{k}, \nu, \widetilde{M}_{k l}\right)$ is a pair of bundle maps $f: A_{k} \rightarrow C_{k}, g: \widetilde{M}_{k l} \rightarrow \widetilde{M}_{k l}$ which are fiberwise isomorphisms of algebras such that the diagram

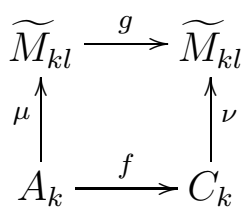

commutes.

Clearly, FABs over $X$ with just defined morphisms form a category $\mathfrak{F} \mathfrak{A} \mathfrak{B}(X)$.

For a continuous map $\varphi: X \rightarrow Y$ we have the natural transformation $\varphi^{*}: \mathfrak{F} \mathfrak{A} \mathfrak{B}(Y) \rightarrow \mathfrak{F} \mathfrak{A} \mathfrak{B}(X)$.

Suppose $(k, l)=1$. Put $\widetilde{\mathcal{M}}_{k l}:=\operatorname{Gr}_{k, l} \times M_{k l}(\mathbb{C})$. Note that there is the tautological $F A B\left(\mathcal{A}_{k, l}, \mu, \widetilde{\mathcal{M}}_{k l}\right)$ over $\operatorname{Gr}_{k, l}$, where $\mu$ is the natural inclusion existing by the definition of $\mathcal{A}_{k, l}$. 
Remark 6. Note once more that there are compact and noncompact versions of the considered theory. In the first case we consider only unitary $k$-subalgebras and for any $x \in \mathrm{Gr}_{k, l}$ the embedding $\left.\mu\right|_{x}:\left(\mathcal{A}_{k, l}\right)_{x} \rightarrow M_{k l}(\mathbb{C})$ is a unitary map (with respect to the standard Hermitian metrics).

Let $\Psi_{k, l}(X)$ be the set of isomorphism classes of FABs of the form $\left(A_{k}, \mu, \widetilde{M}_{k l}\right)$ over $X$. It is clear that for such a FAB there is a classifying map $X \rightarrow \operatorname{Gr}_{k, l}$ (it can be obtained by fixing a trivialization on $\widetilde{M}_{k l}$ ). Furthermore, the following assertion holds.

Proposition 7. ([3], Proposition 1.19) If $\operatorname{dim} X<2 \min \{k, l\}$, then the assignment

$$
\left[X, \operatorname{Gr}_{k, l}\right] \rightarrow \Psi_{k, l}(X), \quad \varphi \mapsto \varphi^{*}\left(\mathcal{A}_{k, l}, \mu, \widetilde{\mathcal{M}}_{k l}\right)
$$

is a bijection.

\subsection{The homogeneous space $\operatorname{Hom}\left(M_{k}(\mathbb{C}), M_{k l}(\mathbb{C})\right)$}

Fix a pair of coprime positive integers $k, l$. Consider the set $\operatorname{Hom}\left(M_{k}(\mathbb{C}), M_{k l}(\mathbb{C})\right)$ of all homomorphisms of matrix algebras that take the unit $1_{M_{k}(\mathbb{C})}=E_{k}$ to the unit $1_{M_{k l}(\mathbb{C})}=$ $E_{k l}$. Since a matrix algebra is a simple ring, we see that any such a homomorphism is an embedding.

There are two natural group actions on the set $\operatorname{Hom}\left(M_{k}(\mathbb{C}), M_{k l}(\mathbb{C})\right)$. First, the group $\mathrm{PGL}_{k}(\mathbb{C})=\operatorname{Aut}\left(M_{k}(\mathbb{C})\right)$ acts on $\operatorname{Hom}\left(M_{k}(\mathbb{C}), M_{k l}(\mathbb{C})\right)$ as follows:

$$
\mathrm{PGL}_{k}(\mathbb{C}) \times \operatorname{Hom}\left(M_{k}(\mathbb{C}), M_{k l}(\mathbb{C})\right) \rightarrow \operatorname{Hom}\left(M_{k}(\mathbb{C}), M_{k l}(\mathbb{C})\right),(g, \vartheta) \mapsto \vartheta \circ g^{-1} .
$$

Secondly, the group $\mathrm{PGL}_{k l}(\mathbb{C})=\operatorname{Aut}\left(M_{k l}(\mathbb{C})\right)$ acts on $\operatorname{Hom}\left(M_{k}(\mathbb{C}), M_{k l}(\mathbb{C})\right)$ in the following way:

$$
\operatorname{PGL}_{k l}(\mathbb{C}) \times \operatorname{Hom}\left(M_{k}(\mathbb{C}), M_{k l}(\mathbb{C})\right) \rightarrow \operatorname{Hom}\left(M_{k}(\mathbb{C}), M_{k l}(\mathbb{C})\right),(g, \vartheta) \mapsto g \circ \vartheta
$$

In particular, we have the corresponding action of the subgroup $\mathrm{PGL}_{k}(\mathbb{C})=\mathrm{PGL}_{k}(\mathbb{C}) \otimes$ $E_{l} \subset \mathrm{PGL}_{k l}(\mathbb{C})$, where by $\otimes$ and $E_{l}$ we denote the Kronecker product of matrices and the unit $l \times l$-matrix respectively.

Note that natural actions (11) and (2) do commute. Indeed, it is a general fact: to given sets $S$ and $R$ and a mapping $f: S \rightarrow R$ one has $(\alpha \circ f) \circ \beta=\alpha \circ(f \circ \beta)$ for all $\alpha \in \Sigma(R), \beta \in \Sigma(S)$, where $\Sigma(T)$ is the group of all bijections of the set $T$.

We also can consider the set $\operatorname{Hom}\left(M_{k}(\mathbb{C}), M_{k l}(\mathbb{C})\right)$ as a $\mathrm{PGL}_{k}(\mathbb{C})$ and $\mathrm{PGL}_{k l}(\mathbb{C})$ invariant subset in the vector space $\operatorname{Hom}_{\text {Vect.sp }}\left(L\left(M_{k}(\mathbb{C})\right), L\left(M_{k l}(\mathbb{C})\right)\right)$, where $L$ is the forgetful functor which to a matrix algebra assigns its underlying vector space. If we choose bases in $L\left(M_{k}(\mathbb{C})\right)$ and $L\left(M_{k l}(\mathbb{C})\right)$, we can identify the latter space with the space of $(k l)^{2} \times k^{2}$-matrices. Then actions (11) and (2) correspond to the right and left multiplication by invertible $k^{2} \times k^{2}$ and $(k l)^{2} \times(k l)^{2}$-matrices respectively.

Now let us describe $\operatorname{Hom}\left(M_{k}(\mathbb{C}), M_{k l}(\mathbb{C})\right)$ as a homogeneous space of the group $\mathrm{PGL}_{k l}(\mathbb{C})$. 
Definition 8. A $k$-frame in $M_{k l}(\mathbb{C})$ is an ordered collection of $k^{2}$ linearly independent matrices $\left\{\alpha_{i, j} \mid 1 \leq i, j \leq k\right\}$ such that $\alpha_{i, j} \alpha_{m, n}=\delta_{j m} \alpha_{i, n}$ for all $1 \leq i, j, m, n \leq k$, where $\delta_{j m}$ is the Kronecker delta-symbol, and $\sum_{1 \leq i \leq k} \alpha_{i, i}=E_{k l}$, where $E_{k l} \in M_{k l}(\mathbb{C})$ is the unit matrix.

Clearly, every such a $k$-frame is a basis in a certain (uniquely determined by the frame) central $k$-subalgebra in $M_{k l}(\mathbb{C})$. For example, there is the "standard" $k$-frame $\left\{e_{i, j} \mid 1 \leq i, j \leq k\right\}$, where $e_{i, j}:=E_{i j} \otimes E_{l}$ is the Kronecker product of a "matrix unit" $E_{i j}$ of order $k$ by the unit $l \times l$ matrix $E_{l}$. It is a frame in the subalgebra $M_{k}(\mathbb{C}) \underset{\mathbb{C}}{\otimes} \mathbb{C} E_{l} \subset M_{k l}(\mathbb{C})$. Applying Noether-Skolem's theorem, we see that all $k$-frames in $M_{k l}(\mathbb{C})$ are conjugate to each other.

Thus, all $k$-frames in $M_{k l}(\mathbb{C})$ form the homogeneous space

$$
\operatorname{Fr}_{k, l}:=\mathrm{PGL}_{k l}(\mathbb{C}) / E_{k} \otimes \mathrm{PGL}_{l}(\mathbb{C})
$$

The notation means that the group $\mathrm{PGL}_{l}(\mathbb{C})$ is embedded into the group $\mathrm{PGL}_{k l}(\mathbb{C})$ by means of the Kronecker product of matrices $X \mapsto E_{k} \otimes X$.

Proposition 9. The space $\operatorname{Hom}\left(M_{k}(\mathbb{C}), M_{k l}(\mathbb{C})\right)$ is a homogeneous space over the group $\mathrm{PGL}_{k l}(\mathbb{C})$ with respect to the action (2). Furthermore, there is an isomorphism of homogeneous spaces $\operatorname{Hom}\left(M_{k}(\mathbb{C}), M_{k l}(\mathbb{C})\right) \cong \operatorname{Fr}_{k, l}$.

Proof. Fix a $k$-frame $\alpha$ in $M_{k}(\mathbb{C})$, i.e. an ordered collection of linearly independent matrices $\left\{\alpha_{i, j} \mid 1 \leq i, j \leq k\right\}, \alpha_{i, j} \in M_{k}(\mathbb{C})$ such that $\alpha_{i, j} \alpha_{r, s}=\delta_{j r} \alpha_{i, s}$. Then we have a one-to-one correspondence between homomorphisms $h \in \operatorname{Hom}\left(M_{k}(\mathbb{C}), M_{k l}(\mathbb{C})\right)$ and $k$-frames $\beta$ in $M_{k l}(\mathbb{C})$ given by $h \leftrightarrow \beta=h(\alpha)$.

In particular, the previous proposition equips the set $\operatorname{Hom}\left(M_{k}(\mathbb{C}), M_{k l}(\mathbb{C})\right)$ with the topology of homogeneous space.

The space $\mathrm{Fr}_{k, l}$ is the total space of the principal $\mathrm{PGL}_{k}(\mathbb{C})$-bundle

$$
\mathrm{Fr}_{k, l} \stackrel{\mathrm{PGL}_{k}(\mathbb{C})}{\longrightarrow} \mathrm{Gr}_{k, l}
$$

The fiber of this bundle over $x \in \mathrm{Gr}_{k, l}$ consists of all those $k$-frames that are contained in the $k$-subalgebra $M_{k, x} \subset M_{k l}(\mathbb{C})$ corresponding to $x$. The tautological $M_{k}(\mathbb{C})$-bundle $\mathcal{A}_{k, l}$ over $\mathrm{Gr}_{k, l}$ is associated with this principal bundle. Thus, the homogeneous space $\mathrm{Fr}_{k, l}$ can be treated as the total space of the principal $\mathrm{PGL}_{k}(\mathbb{C})$-bundle $\operatorname{Prin}\left(\mathcal{A}_{k, l}\right) \rightarrow$ $\mathrm{Gr}_{k, l}$.

Remark 10. There is also a unitary analog of this notion. Clearly, the set of all unitary $k$-frames in $M_{k l}(\mathbb{C})$ (with respect to the standard Hermitian metric $\langle A, B\rangle=\operatorname{tr}\left(A \bar{B}^{t}\right)$ on $\left.M_{k l}(\mathbb{C})\right)$ is the homogeneous space $\mathrm{Fr}_{k, l}:=\mathrm{PU}(k l) / E_{k} \otimes \mathrm{PU}(l)$. It is the total space of the following principal bundle

$$
\mathrm{Fr}_{k, l} \stackrel{\mathrm{PU}(k)}{\longrightarrow} \mathrm{Gr}_{k, l}
$$


over the (compact) matrix Grassmannian $\mathrm{Gr}_{k, l}$. The fiber of this bundle over $x \in \mathrm{Gr}_{k, l}$ consists of all those unitary $k$-frames that are contained in the $k$-subalgebra $M_{k, x} \subset$ $M_{k l}(\mathbb{C})$ corresponding to $x$ (recall that points $x \in \mathrm{Gr}_{k, l}$ correspond to unitary central $k$-subalgebras in $M_{k l}(\mathbb{C})$ ). The tautological $M_{k}(\mathbb{C})$-bundle $\mathcal{A}_{k, l}$ over $\operatorname{Gr}_{k, l}$ is associated with this principal bundle.

Now note that there is a canonical FAB over $\operatorname{Hom}\left(M_{k}(\mathbb{C}), M_{k l}(\mathbb{C})\right)=\mathrm{Fr}_{k, l}$ whose core (which is a trivial $M_{k}(\mathbb{C})$-bundle equipped with the canonical trivialization) is defined as the subspace

$\left\{(h, h(T)) \mid h \in \operatorname{Hom}\left(M_{k}(\mathbb{C}), M_{k l}(\mathbb{C})\right), T \in M_{k}(\mathbb{C})\right\} \subset \operatorname{Hom}\left(M_{k}(\mathbb{C}), M_{k l}(\mathbb{C})\right) \times M_{k l}(\mathbb{C})$.

Obviously, this FAB is the pull-back of the tautological FAB $\left(\mathcal{A}_{k, l}, \mu, \widetilde{\mathcal{M}}_{k l}\right)$ under the projection $\mathrm{Fr}_{k, l} \rightarrow \mathrm{Gr}_{k, l}$. Note that the considered $\mathrm{FAB}$ over $\operatorname{Hom}\left(M_{k}(\mathbb{C}), M_{k l}(\mathbb{C})\right)$ is not trivial (see Definition 13 below) although its core is. The reason of this is that the embedding $\mu$ is "twisted".

Obviously, the assignment $M_{k}(\mathbb{C}) \mapsto \operatorname{Hom}\left(M_{k}(\mathbb{C}), M_{k l}(\mathbb{C})\right)$ is functorial and therefore it can be transferred to locally trivial bundles with fiber $M_{k}(\mathbb{C})$. Thus, to any locally trivial $M_{k}(\mathbb{C})$-bundle $A_{k}$ over $X$ we assign the locally trivial $\operatorname{Hom}\left(M_{k}(\mathbb{C}), M_{k l}(\mathbb{C})\right)$ bundle (over the same base $X$ ). The obtained bundle we denote by $\mathbf{H}_{k, l}\left(A_{k}\right)$. In particular, $\mathbf{H}_{k, l}\left(M_{k}(\mathbb{C})\right) \cong \operatorname{Fr}_{k, l}$, where $M_{k}(\mathbb{C})$ is regarded as a trivial bundle over a point.

In terms of structure groups, the bundle $\mathbf{H}_{k, l}\left(A_{k}\right)$ is associated with the same principal $\mathrm{PGL}_{k}(\mathbb{C})$-bundle as $A_{k}$, using the action (11).

\subsection{The $\operatorname{Hom}\left(M_{k}(\mathbb{C}), M_{k l}(\mathbb{C})\right)$-bundle $\mathbf{H}_{k, l}\left(A_{k}^{\text {univ }}\right) \stackrel{p}{\rightarrow} \operatorname{BPU}(k)$}

By $A_{k}^{\text {univ }}$ denote the universal $M_{k}(\mathbb{C})$-bundle over $\mathrm{BPU}(k)$ (it is a locally trivial bundle with the structure group $\left.\mathrm{PU}(k) \simeq \mathrm{PGL}_{k}(\mathbb{C})=\operatorname{Aut}\left(M_{k}(\mathbb{C})\right)\right)$. In this subsection we study the $\operatorname{Hom}\left(M_{k}(\mathbb{C}), M_{k l}(\mathbb{C})\right)$-bundle $\mathbf{H}_{k, l}\left(A_{k}^{\text {univ }}\right) \stackrel{p}{\rightarrow} \operatorname{BPU}(k)$.

Let $t_{k, l}: \mathrm{Gr}_{k, l} \rightarrow \mathrm{BPU}(k)$ be the classifying map for $\mathcal{A}_{k, l}$ as a bundle with the structure group $\mathrm{PGL}_{k}(\mathbb{C})$. Recall that the mapping $t_{k, l}$ can be considered as a fibration in Homotopy Category.

First note that there is the canonical embedding $p^{*}\left(A_{k}^{\text {univ }}\right) \stackrel{\widetilde{\mu}}{\hookrightarrow} \mathbf{H}_{k, l}\left(A_{k}^{\text {univ }}\right) \times M_{k l}(\mathbb{C})$ given by $(h, a) \mapsto(h, h(a))$, where $a \in\left(A_{k}^{\text {univ }}\right)_{x}, h \in \operatorname{Hom}\left(\left(A_{k}^{\text {univ }}\right)_{x}, M_{k l}(\mathbb{C})\right)$. Thus the canonical FAB $\left(p^{*}\left(A_{k}^{\text {univ }}\right), \widetilde{\mu}, \mathbf{H}_{k, l}\left(A_{k}^{\text {univ }}\right) \times M_{k l}(\mathbb{C})\right)$ over $\mathbf{H}_{k, l}\left(A_{k}^{\text {univ }}\right)$ is defined.

Theorem 11. The total space $\mathbf{H}_{k, l}\left(A_{k}^{\text {univ }}\right)$ is homotopy equivalent to the matrix Grassmannian $\mathrm{Gr}_{k, l}$. Moreover, the bundle projection $\mathbf{H}_{k, l}\left(A_{k}^{\text {univ }}\right) \stackrel{p}{\rightarrow} \mathrm{BPU}(k)$ can be identified with the classifying map $t_{k, l}$ under this homotopy equivalence.

This theorem is a consequence of the following proposition. 
Let $X$ be a finite $C W$-complex. Consider the set $S_{k, l}(X)$ of equivalence classes of FABs of the form $\left(A_{k}, \mu, \widetilde{M}_{k l}\right)$ over $X$ with respect to the following equivalence relation:

$$
\left(A_{k}, \mu, \widetilde{M}_{k l}\right) \sim\left(A_{k}^{\prime}, \mu^{\prime}, \widetilde{M}_{k l}\right) \Leftrightarrow
$$

there is a fiberwise homotopy $M: A_{k} \times I \rightarrow \widetilde{M}_{k l}$ such that

$$
\left.M\right|_{A_{k} \times\{0\}}=\mu,\left.M\right|_{A_{k} \times\{1\}}: A_{k} \cong \mu^{\prime}\left(A_{k}^{\prime}\right) \subset \widetilde{M}_{k l}
$$

(in particular, $A_{k} \cong A_{k}^{\prime}$ and moreover $\left(A_{k}, \mu, \widetilde{M}_{k l}\right) \cong\left(A_{k}^{\prime}, \mu^{\prime}, \widetilde{M}_{k l}\right)$ ).

Proposition 12. The spaces $\mathrm{Gr}_{k, l}$ and $\mathbf{H}_{k, l}\left(A_{k}^{\text {univ }}\right)$ both represent the homotopy functor $X \mapsto S_{k, l}(X)$.

Proof. For $\operatorname{Gr}_{k, l}$ this is clear. Consider $\mathbf{H}_{k, l}\left(A_{k}^{\text {univ }}\right)$. Suppose $\left(A_{k}, \mu, \widetilde{M}_{k l}\right) \sim$ $\left(A_{k}^{\prime}, \mu^{\prime}, \widetilde{M}_{k l}\right)$ and $\varphi_{0}, \varphi_{1}: X \quad \rightarrow \quad \mathbf{H}_{k, l}\left(A_{k}^{\text {univ }}\right)$ are classifying maps for $\left(A_{k}, \mu, \widetilde{M}_{k l}\right), \quad\left(A_{k}^{\prime}, \mu^{\prime}, \widetilde{M}_{k l}\right)$ respectively. We have to construct a homotopy $\Phi$ between $\varphi_{0}$ and $\varphi_{1}$.

Put $\bar{\varphi}_{i}:=p \circ \varphi_{i}, i=0,1$, where $p: \mathbf{H}_{k, l}\left(A_{k}^{\text {univ }}\right) \rightarrow \operatorname{BPU}(k)$ is the projection. Since $A_{k} \cong A_{k}^{\prime}$, we have a homotopy $\bar{\Phi}: X \times I \rightarrow \operatorname{BPU}(k)$ between $\bar{\varphi}_{0}$ and $\bar{\varphi}_{1}$. This (together with the covering homotopy property) shows that without loss of generality we can assume that $\bar{\varphi}_{0}=\bar{\varphi}_{1}=: \bar{\varphi}$. In other words, $A_{k}=A_{k}^{\prime}=\bar{\varphi}^{*}\left(A_{k}^{\text {univ }}\right)$ and $M$ defines a homotopy between $\mu: A_{k} \rightarrow \widetilde{M}_{k l}$ and $\mu^{\prime}: A_{k} \rightarrow \widetilde{M}_{k l}$ (indeed, such a homotopy can be chosen as the composition $M \circ \alpha$, where $\alpha: A_{k} \cong A_{k}^{\prime}$ is an arbitrary isomorphism). We have the bundle map $\widetilde{\varphi}: \mathbf{H}_{k, l}\left(A_{k}\right) \rightarrow \mathbf{H}_{k, l}\left(A_{k}^{\text {univ }}\right)$ which covers $\bar{\varphi}: X \rightarrow \operatorname{BPU}(k)$. There is the natural one-to-one correspondence between sections of the bundle $\mathbf{H}_{k, l}\left(A_{k}\right) \rightarrow X$ and embeddings $A_{k} \hookrightarrow \widetilde{M}_{k l}=X \times M_{k l}(\mathbb{C})$ and also between corresponding homotopies. Let $\sigma_{i}, i=0,1$ be the sections corresponding to $\mu, \mu^{\prime}$ respectively. Therefore the homotopy $M$ gives us a homotopy $\varsigma$ between $\sigma_{0}$ and $\sigma_{1}$. Finally, the composite map $\widetilde{\varphi} \circ \varsigma: X \times I \rightarrow \mathbf{H}_{k, l}\left(A_{k}^{\text {univ }}\right)$ is the required homotopy between the classifying maps $\varphi_{0}: X \rightarrow \mathbf{H}_{k, l}\left(A_{k}^{\text {univ }}\right)$ and $\varphi_{1}: X \rightarrow \mathbf{H}_{k, l}\left(A_{k}^{\text {univ }}\right)$.

Now the converse assertion is clear.

Since the both spaces represent the same homotopy functor, there is a homotopy equivalence $\mathbf{H}_{k, l}\left(A_{k}^{\text {univ }}\right) \simeq \mathrm{Gr}_{k, l}$.

Thus, we see that a lifting in the bundle $\mathbf{H}_{k, l}\left(A_{k}^{\text {univ }}\right) \rightarrow \mathrm{BPU}(k)$ and an embedding of a locally trivial algebra bundle $A_{k}$ into a trivial one (with fiber $M_{k l}(\mathbb{C})$ ) are the same things. This will be useful for the study of obstructions for such an embedding in Subsection 3.2.

Now let us describe the homotopy equivalence $\mathrm{Gr}_{k, l} \simeq \mathbf{H}_{k, l}\left(A_{k}^{\text {univ }}\right)$ more explicitly. Obviously, the canonical embedding

$$
p^{*}\left(A_{k}^{\text {univ }}\right) \stackrel{\widetilde{\mu}}{\hookrightarrow} \mathbf{H}_{k, l}\left(A_{k}^{\text {univ }}\right) \times M_{k l}(\mathbb{C})
$$


determines the map $\varphi: \mathbf{H}_{k, l}\left(A_{k}^{\text {univ }}\right) \rightarrow \operatorname{Gr}_{k, l}$ such that $\varphi^{*}\left(\mathcal{A}_{k, l}, \mu, \mathrm{Gr}_{k, l} \times M_{k l}(\mathbb{C})\right)=$ $\left(p^{*}\left(A_{k}^{\text {univ }}\right), \widetilde{\mu}, \mathbf{H}_{k, l}\left(A_{k}^{\text {univ }}\right) \times M_{k l}(\mathbb{C})\right)$, where $\left(\mathcal{A}_{k, l}, \mu, \mathrm{Gr}_{k, l} \times M_{k l}(\mathbb{C})\right)$ is the tautological FAB over $\mathrm{Gr}_{k, l}$. (Indeed, $\varphi$ can be defined as follows. To a given $h \in \mathbf{H}_{k, l}\left(A_{k}^{\text {univ }}\right)$ the image $\widetilde{\mu}\left(p^{*}\left(A_{k}^{\text {univ }}\right)_{h}\right) \subset M_{k l}(\mathbb{C}) \cong\{h\} \times M_{k l}(\mathbb{C})$ is a subalgebra isomorphic to $M_{k}(\mathbb{C})$. We put $\varphi(h)=\alpha \in \mathrm{Gr}_{k, l}$, where $\alpha$ is the point corresponding to the $k$-subalgebra $\widetilde{\mu}\left(p^{*}\left(A_{k}^{\text {univ }}\right)_{h}\right) \subset M_{k l}(\mathbb{C})$.) Since $t_{k, l}: \mathrm{Gr}_{k, l} \rightarrow \mathrm{BPU}(k)$ is a classifying map for $\mathcal{A}_{k, l}$, we have $p \simeq t_{k, l} \circ \varphi$.

From the other hand, we have the fibration

$$
\mathrm{Fr}_{k, l} \rightarrow \mathrm{Gr}_{k, l} \stackrel{t_{k, l}}{\longrightarrow} \mathrm{BPU}(k) .
$$

The embedding $\mu: \mathcal{A}_{k, l} \hookrightarrow \mathrm{Gr}_{k, l} \times M_{k l}(\mathbb{C})$ gives us a lifting $\psi: \mathrm{Gr}_{k, l} \rightarrow \mathbf{H}_{k, l}\left(A_{k}^{\text {univ }}\right)$ of $t_{k, l}$. (Indeed, for $\alpha \in \operatorname{Gr}_{k, l}, t_{k, l}(\alpha)=x$ we put $\psi(\alpha)=h$, where $h \in\left(\mathbf{H}_{k, l}\left(A_{k}^{\text {univ }}\right)\right)_{x}=$ $\operatorname{Hom}\left(\left(A_{k}^{\text {univ }}\right)_{x}, M_{k l}(\mathbb{C})\right)=\operatorname{Hom}\left(\left(\mathcal{A}_{k, l}\right)_{\alpha}, M_{k l}(\mathbb{C})\right)$ is the homomorphism defined by $\left.\left.\mu\right|_{\alpha}\right)$. Clearly, $\psi^{*}\left(p^{*}\left(A_{k}^{\text {univ }}\right), \widetilde{\mu}, \mathbf{H}_{k, l}\left(A_{k}^{\text {univ }}\right) \times M_{k l}(\mathbb{C})\right)=\left(\mathcal{A}_{k, l}, \mu, \widetilde{\mathcal{M}}_{k l}\right)$. In particular, $p \circ \psi \simeq$ $t_{k, l}$.

We have already observed that since the both spaces $\mathbf{H}_{k, l}\left(A_{k}^{\text {univ }}\right)$ and $\mathrm{Gr}_{k, l}$ represent the same homotopy functor, they are homotopy equivalent to each other. Moreover it is easy to see that the above constructed maps $\varphi: \mathbf{H}_{k, l}\left(A_{k}^{\text {univ }}\right) \rightarrow \mathrm{Gr}_{k, l}$ and $\psi: \mathrm{Gr}_{k, l} \rightarrow$ $\mathbf{H}_{k, l}\left(A_{k}^{\text {univ }}\right)$ are the desired homotopy equivalences, $\psi \circ \varphi \simeq \operatorname{id}_{\mathbf{H}_{k, l}\left(A_{k}^{\text {univ }}\right)}, \varphi \circ \psi \simeq$ $\operatorname{id}_{\mathrm{Gr}_{k, l}}$.

Thus, we can identify the fibrations (in Homotopy Category)

$$
\mathrm{Fr}_{k, l} \rightarrow \mathrm{Gr}_{k, l} \stackrel{t_{k, l}}{\longrightarrow} \mathrm{BPU}(k)
$$

and

$$
\mathrm{Fr}_{k, l} \rightarrow \mathbf{H}_{k, l}\left(A_{k}^{\text {univ }}\right) \stackrel{p}{\rightarrow} \operatorname{BPU}(k)
$$

\section{The functor represented by the $H$-space $\mathrm{Gr}$}

In this section we give a brief survey of the stable theory of floating algebra bundles. We do this because this theory is needed to motivate the definition of the generalized Brauer group in Subsection 3.3 (see for example Theorem 43 and its corollary). For more details see [3], Ch.2.

\subsection{The stable equivalence of FABs}

Define the product $\circ$ of two FABs over $X\left(A_{k}, \mu, \widetilde{M}_{k l}\right),\left(B_{m}, \nu, \widetilde{M}_{m n}\right)$ such that $(k m, l n)=1$ as

$$
\left(A_{k}, \mu, \widetilde{M}_{k l}\right) \circ\left(B_{m}, \nu, \widetilde{M}_{m n}\right):=\left(A_{k} \otimes B_{m}, \mu \otimes \nu, \widetilde{M}_{k l} \otimes \widetilde{M}_{m n}\right)
$$

(notice that $\widetilde{M}_{k l} \otimes \widetilde{M}_{m n}=\widetilde{M}_{k l m n}$ ). 
Definition 13. A FAB of the form $\left(\widetilde{M}_{k}, \tau, \widetilde{M}_{k l}\right)$ is called trivial if the map $\tau: \widetilde{M}_{k} \rightarrow$ $\widetilde{M}_{k l}$ is the following:

$$
X \times M_{k}(\mathbb{C}) \rightarrow X \times M_{k l}(\mathbb{C}), \quad(x, T) \mapsto\left(x, T \otimes E_{l}\right) \text { for all } x \in X
$$

(under some choice of trivializations on $\widetilde{M}_{k}$ and $\widetilde{M}_{k l}$ ), where $E_{l}$ is the unit $l \times l$-matrix and $T \otimes E_{l}$ denotes the Kronecker product of matrices. In other words, the bundle $\widetilde{M}_{k}$ is embedded into $\widetilde{M}_{k l}$ as a fixed subalgebra.

Definition 14. Two FABs $\left(A_{k}, \mu, \widetilde{M}_{k l}\right)$ and $\left(B_{m}, \nu, \widetilde{M}_{m n}\right)$ over $X$ are said to be stable equivalent if there is a sequence of pairs $\left\{t_{i}, u_{i}\right\} \in \mathbb{N}^{2}, 1 \leq i \leq s$ such that

- $\left\{t_{1}, u_{1}\right\}=\{k, l\},\left\{t_{s}, u_{s}\right\}=\{m, n\}$;

- $\left(t_{i} t_{i+1}, u_{i} u_{i+1}\right)=1$ if $s>1,1 \leq i \leq s-1$,

and a corresponding sequence of FABs $\left(A_{t_{i}}, \mu_{i}, \widetilde{M}_{t_{i} u_{i}}\right)$ over $X$ such that

- $\left(A_{t_{1}}, \mu_{1}, \widetilde{M}_{t_{1} u_{1}}\right)=\left(A_{k}, \mu, \widetilde{M}_{k l}\right),\left(A_{t_{s}}, \mu_{s}, \widetilde{M}_{t_{s} u_{s}}\right)=\left(B_{m}, \nu, \widetilde{M}_{m n}\right)$;

- $\left(A_{t_{i}}, \mu_{i}, \widetilde{M}_{t_{i} u_{i}}\right) \circ\left(\widetilde{M}_{t_{i+1}}, \tau, \widetilde{M}_{t_{i+1} u_{i+1}}\right) \cong\left(A_{t_{i+1}}, \mu_{i+1}, \widetilde{M}_{t_{i+1} u_{i+1}}\right) \circ\left(\widetilde{M}_{t_{i}}, \tau, \widetilde{M}_{t_{i} u_{i}}\right)$, where $1 \leq i \leq s-1$ and $\left(\widetilde{M}_{t_{i}}, \tau, \widetilde{M}_{t_{i} u_{i}}\right)$ are trivial FABs.

By $\widetilde{\mathrm{AB}}^{1}(X)$ denote the set of stable equivalence classes of FABs over $X$.

The following theorem justifies the previous definition. Note that a homomorphism of central algebras $M_{k l}(\mathbb{C}) \hookrightarrow M_{k l m n}(\mathbb{C})$ induces the corresponding map of matrix Grassmannians $i_{k, l ; m, n}: \mathrm{Gr}_{k, l} \rightarrow \mathrm{Gr}_{k m, l n}$; the direct limits below are taken over such maps.

Theorem 15. ( [3], Proposition 2.1) 1) For all sequences of pairs of positive integers $\left\{k_{j}, l_{j}\right\}_{j \in \mathbb{N}}$ such that

$$
\text { (i) } \quad k_{j}, l_{j} \rightarrow \infty ; \quad \text { (ii) } k_{j}\left|k_{j+1}, l_{j}\right| l_{j+1} ; \quad \text { (iii) }\left(k_{j}, l_{j}\right)=1
$$

for every $j$, the corresponding direct limits $\underset{\vec{j}}{\lim } \mathrm{Gr}_{k_{j}, l_{j}}$ are homotopy-equivalent. This unique homotopy type we denote by $\mathrm{Gr}$.

2) $\mathrm{Gr}$ is a classifying space for stable equivalence classes of FABs over a finite $C W$ complex $X$. In other words, the functor $X \mapsto \widetilde{\mathrm{AB}}^{1}(X)$ from the homotopy category of finite $C W$-complexes to the category $\mathfrak{S e t s}$ is represented by the space $\mathrm{Gr}$.

The proof is based on Proposition 7 and on the following lemma.

Lemma 16. If $(k m, \ln )=1$, then the embedding

$$
i_{k, l ; m, n}: \mathrm{Gr}_{k, l} \rightarrow \mathrm{Gr}_{k m, l n}
$$

is a homotopy equivalence in dimensions $<2 \min \{k, l\}$. 
Therefore for any finite $C W$-complex $X, \operatorname{dim}(X)<2 \min \{k, l, m, n\}$ and for any map $\varphi_{k m, l n}: X \rightarrow \mathrm{Gr}_{k m, l n}$ there are maps $\varphi_{k, l}: X \rightarrow \operatorname{Gr}_{k, l}$ and $\varphi_{m, n}: X \rightarrow$ $\mathrm{Gr}_{m, n}$ such that $i_{k, l ; m, n} \circ \varphi_{k, l} \simeq \varphi_{k m, l n} \simeq i_{m, n ; k, l} \circ \varphi_{m, n}$. Note also that $i_{k, l ; m, n}^{*}\left(\mathcal{A}_{k m, l n}, \mu, \widetilde{\mathcal{M}}_{k l m n}\right)=\left(\mathcal{A}_{k, l}, \mu, \widetilde{\mathcal{M}}_{k l}\right) \circ\left(\widetilde{\mathcal{M}}_{m}, \tau, \widetilde{\mathcal{M}}_{m n}\right)$, whence the stable equivalence relation.

\subsection{The group structure}

Let $\left(A_{k}, \mu, \widetilde{M}_{k l}\right)$ be a FAB over $X$. By $\left[\left(A_{k}, \mu, \widetilde{M}_{k l}\right)\right]$ we denote its stable equivalence class (with respect to the equivalence relation defined in the previous subsection). Define the product $\diamond$ of two classes $\left[\left(A_{k}, \mu, \widetilde{M}_{k l}\right)\right],\left[\left(B_{m}, \nu, \widetilde{M}_{m n}\right)\right]$ for $(k m, l n)=1$ as

$$
\begin{gathered}
{\left[\left(A_{k}, \mu, \widetilde{M}_{k l}\right)\right] \diamond\left[\left(B_{m}, \nu, \widetilde{M}_{m n}\right)\right]=\left[\left(A_{k}, \mu, \widetilde{M}_{k l}\right) \circ\left(B_{m}, \nu, \widetilde{M}_{m n}\right)\right]} \\
=\left[\left(A_{k} \otimes B_{m}, \mu \otimes \nu, \widetilde{M}_{k l m n}\right)\right] .
\end{gathered}
$$

Clearly, this product is well defined. The following lemma allows us to reject the restriction $(k m, l n)=1$.

Lemma 17. For any pair $\{k, l\}$ such that (i) $(k, l)=1$, (ii) $2 \min \{k, l\} \geq \operatorname{dim} X$, any stable equivalence class of FABs over $X$ has a representative of the form $\left(A_{k}, \mu, \widetilde{M}_{k l}\right)$.

Clearly, the product $\diamond$ is associative, commutative, and has identity element $\left[\left(\widetilde{M}_{k}, \tau, \widetilde{M}_{k l}\right)\right]$, where $\left(\widetilde{M}_{k}, \tau, \widetilde{M}_{k l}\right)$ is a trivial FAB. Moreover, for any class $\left[\left(A_{k}, \mu, \widetilde{M}_{k l}\right)\right]$ there exists the inverse element. In order to find it, let us recall the following fact. The centralizer $\mathrm{Z}_{P}(Q)$ of a central simple subalgebra $Q$ in a central simple algebra $P$ (over some field $\mathbb{K}$ ) is a central simple subalgebra again, moreover, the equality $P=Q \underset{\mathbb{K}}{\otimes} \mathrm{Z}_{P}(Q)$ holds. Therefore by taking centralizers for every fiber of the subbundle $A_{k}$ in $\widetilde{M}_{k l}$, we obtain the complementary subbundle $B_{l}$ with fiber $M_{l}(\mathbb{C})$ together with its embedding $\nu: B_{l} \hookrightarrow \widetilde{M}_{k l}$ into the trivial bundle. Moreover, $A_{k} \otimes B_{l}=\widetilde{M}_{k l}$. It is not hard to prove that $\left[\left(B_{l}, \nu, \widetilde{M}_{k l}\right)\right]$ is the inverse element for $\left[\left(A_{k}, \mu, \widetilde{M}_{k l}\right)\right]$. Thus, the functor $X \mapsto \widetilde{\mathrm{AB}}^{1}(X)$ takes values in the category of Abelian groups $\mathfrak{A} \mathfrak{b}$.

Proposition 18. (3], Theorem 2.5) The space Gr can be equipped with an H-space structure such that there is a natural equivalence between $X \mapsto[X, \mathrm{Gr}]$ and $X \mapsto$ $\widetilde{\mathrm{AB}}^{1}(X)$ as functors to the category $\mathfrak{A} \mathfrak{b}$.

\subsection{Some properties of FAB's core}

Recall that a locally trivial $\operatorname{Aut}\left(M_{k}(\mathbb{C})\right) \cong \mathrm{PGL}_{k}(\mathbb{C})$ (or $\left.\mathrm{PU}(k)\right)$-bundle $A_{k}$ is called a core of a $\operatorname{FAB}\left(A_{k}, \mu, \widetilde{M}_{k l}\right)$ (if such a FAB exists, of course). We mention its properties because they will play an important role in the definition of the generalized Brauer group in Subsection 3.3. 
Lemma 19. If $A_{k}$ is the core of some $\operatorname{FAB}\left(A_{k}, \mu, \widetilde{M}_{k l}\right)$, then its structure group can be reduced from Aut $M_{k}(\mathbb{C}) \cong \mathrm{PGL}_{k}(\mathbb{C})$ to $\mathrm{SL}_{k}(\mathbb{C})$ (or equivalent from $\mathrm{PU}(k)$ to $\mathrm{SU}(k))$.

Proof. Indeed, since $\rho_{k l}=\rho_{k} \times \rho_{l}$ for $(k, l)=1$, where $\rho_{n}$ is the group of $n$th degree roots of unity, we have

$$
\mathrm{Gr}_{k, l}=\mathrm{PU}(k l) / \mathrm{PU}(k) \otimes \mathrm{PU}(l)=\mathrm{SU}(k l) / \mathrm{SU}(k) \otimes \mathrm{SU}(l)
$$

The following lemma describes a characteristic property of cores.

Lemma 20. ([3], Lemma 2.7) Let $X$ be a finite $C W$-complex. Suppose $\operatorname{dim} X \leq$ $2 \min \{k, m\}$; then the following conditions are equivalent:

- $A_{k}$ is the core of some FAB over $X$;

- for arbitrary $m$ such that $2 m \geq \operatorname{dim} X$ there is a bundle $B_{m}$ with fiber $M_{m}(\mathbb{C})$ such that $A_{k} \otimes \widetilde{M}_{m} \cong B_{m} \otimes \widetilde{M}_{k}$;

- $A_{k} \otimes \widetilde{M}_{m} \cong B_{m} \otimes \widetilde{M}_{k}$ for some locally trivial bundle $B_{m}$ with fiber $M_{m}(\mathbb{C})$, where $(k, m)=1$.

Moreover, for any pair of bundles $A_{k}, B_{m}$ such that $(k, m)=1$ and $A_{k} \otimes \widetilde{M}_{m} \cong B_{m} \otimes \widetilde{M}_{k}$, there exists a unique stable equivalence class of FABs over $X$ which has (for sufficiently large $n,(k m, n)=1)$ FABs of the forms $\left(A_{k}, \mu, \widetilde{M}_{k n}\right),\left(B_{m}, \nu, \widetilde{M}_{m n}\right)$ as representatives (for some embeddings $\mu, \nu$ ).

\subsection{Localization}

Let $X$ be a finite $C W$-complex, $k \geq 2$ a fixed integer. The set of isomorphism classes of bundles of the form $A_{k^{m}}$ (for arbitrary $m \in \mathbb{N}$ ) over $X$ with fiber $M_{k^{m}}(\mathbb{C}$ ) is a monoid with respect to the operation $\otimes\left(\right.$ with the identity element $\left.M_{k^{0}}(\mathbb{C}) \cong \mathbb{C}\right)$.

Let us consider the following equivalence relation

$$
A_{k^{m}} \sim B_{k^{n}} \Longleftrightarrow \exists r, s \in \mathbb{N} \text { such that } A_{k^{m}} \otimes \widetilde{M}_{k^{r}} \cong B_{k^{n}} \otimes \widetilde{M}_{k^{s}}
$$

$(\Rightarrow m+r=n+s)$. The set of equivalence classes $\left[A_{k^{m}}\right]$ of such bundles is a group with respect to the operation induced by $\otimes$. This group we denote by $\widetilde{\mathrm{AB}}^{k}(X)$.

Let us consider the direct limit $\underset{n}{\lim } \operatorname{BPU}\left(k^{n}\right)$ with respect to the maps induced by

$$
\begin{array}{cl}
\mathrm{PU}\left(k^{n}\right) & \hookrightarrow \mathrm{PU}\left(k^{n+1}\right), \\
A & \mapsto E_{k} \otimes A .
\end{array}
$$

Clearly, the functor $X \mapsto \widetilde{\mathrm{AB}}^{k}(X)$ is represented by $\underset{n}{\lim } \operatorname{BPU}\left(k^{n}\right)$. 
According to Lemma 17, for any stable equivalence class of FABs over $X$ there is a representative of the form $\left(A_{k^{m}}, \mu, \widetilde{M}_{(k l)^{m}}\right),(k, l)=1$. Therefore for any $k$ we have the group homomorphism

$$
\widetilde{\mathrm{AB}}^{1}(X) \rightarrow \widetilde{\mathrm{AB}}^{k}(X), \quad\left[\left(A_{k^{m}}, \mu, \widetilde{M}_{(k l)^{m}}\right)\right] \mapsto\left[A_{k^{m}}\right]
$$

induced by the following map of the direct limits

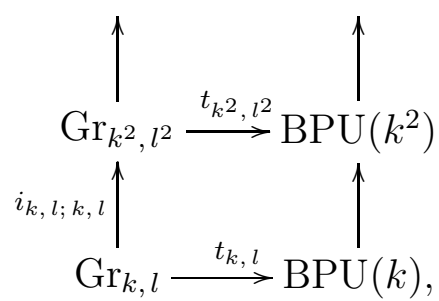

where $t_{k^{2}, l^{2}}$ and $t_{k, l}$ are classifying maps for the cores $\mathcal{A}_{k, l}$ and $\mathcal{A}_{k^{2}, l^{2}}$ as $\mathrm{PU}(k)$ and $\mathrm{PU}\left(k^{2}\right)$-bundles, respectively.

The kernel of the homomorphism $\widetilde{\mathrm{AB}}^{1}(X) \rightarrow \widetilde{\mathrm{AB}}^{k}(X)$ is just the $k$-torsion subgroup in $\widetilde{\mathrm{AB}}^{1}(X)$.

Set $t_{k}:=\underset{r}{\lim } t_{k^{r}, l^{r}}: \operatorname{Gr} \rightarrow \underset{r}{\lim } \operatorname{BPU}\left(k^{r}\right)=: \operatorname{BPU}\left(k^{\infty}\right)$. Then $t_{k}$ is the composition of the localization map $\left([12]\right.$, Ch.2) $\widetilde{t}_{k}: \operatorname{Gr} \rightarrow \underset{r}{\lim } \operatorname{BSU}\left(k^{r}\right)=\operatorname{BSU}\left[\frac{1}{k}\right]$ and the natural map $\lim _{\vec{r}} \mathrm{BSU}\left(k^{r}\right) \rightarrow \underset{r}{\lim _{\vec{r}}} \mathrm{BPU}\left(k^{r}\right)$ induced by the group epimorphisms $\mathrm{SU}\left(k^{r}\right) \rightarrow \mathrm{PU}\left(k^{r}\right)$ with the kernels $\left\{\lambda E_{k^{r}} \mid \lambda^{k^{r}}=1\right\} \cong \rho_{k^{r}}$.

\subsection{Relation between $\widetilde{\mathrm{AB}}^{1}$ and $\widetilde{\mathrm{KSU}}-$ theory}

Recall that $\mathrm{BSU}_{\otimes}$ is the space $\mathrm{BSU}$ with the structure of $H$-space related to the tensor product of virtual SU-bundles of virtual dimension 1.

Theorem 21. ([3], Theorem 2.17) There is an $H$-space isomorphism $\mathrm{Gr} \cong \mathrm{BSU}_{\otimes}$.

By $\widetilde{\mathrm{KSU}}(X)$ denote the reduced $\mathrm{K}$-functor constructed by means of SU-bundles over $X$. Recall that $\widetilde{\mathrm{KSU}}(X)$ is a ring with the multiplication induced by the tensor product of bundles.

The previous theorem claims that the group $\widetilde{\mathrm{AB}}^{1}(X)$ is isomorphic to the multiplicative group of the ring $\widehat{\mathrm{KSU}}(X)$, i.e. the group (because $X$ is a finite $C W$-complex) of elements of $\widetilde{\mathrm{KSU}}(X)$ with respect to the operation $\xi * \eta=\xi+\eta+\xi \eta(\xi, \eta \in \widetilde{\mathrm{KSU}}(X)$, i.e. $\xi, \eta$ are of virtual dimension 0$)$.

This gives us a geometric description of the $H$-structure on $\mathrm{BSU}_{\otimes}$. For example, the construction of the inverse stable equivalence class $\left[\left(B_{m}, \nu, \widetilde{M}_{m n}\right)\right]$ for a given one $\left[\left(A_{k}, \mu, \widetilde{M}_{k l}\right)\right]$ is closely connected with taking centralizer for a subalgebra in a fixed matrix algebra. 
Although the theory of floating algebra bundles leads to the theory which can also be described in the classical terms, the obtained geometric approach might be applicable in other branches of Topology (for example, in Cobordism theory, cf. [4], [5]).

\subsection{A U-version}

Consider the canonical map $\mathrm{BU}(k) \rightarrow \mathrm{BPU}(k)$ induced by the group homomorphism $\mathrm{U}(k) \rightarrow \mathrm{PU}(k)$. By $\widehat{\mathrm{Gr}}_{k, l}$ denote the total space of the $\mathrm{Fr}_{k, l}$-fibration (recall that $\mathrm{Fr}_{k, l}$ denotes the space of (unitary) $k$-frames in $M_{k l}(\mathbb{C})$, see Subsection [1.3) induced by the fibration $\mathrm{Gr}_{k, l} \stackrel{\mathrm{Fr}_{k, l}}{\longrightarrow} \mathrm{BPU}(k)$ and the map $\mathrm{BU}(k) \rightarrow \mathrm{BPU}(k)$ (as ever, the integers $k, l$ are assumed to be coprime), i.e. the fiber product

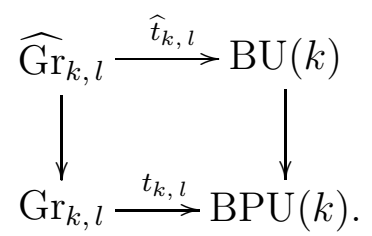

It follows easily that there is a $\mathbb{C} P^{\infty}$-fibration $\widehat{\mathrm{Gr}}_{k, l} \rightarrow \mathrm{Gr}_{k, l}$.

Remark 22. Let us give a description of $\widehat{\operatorname{Gr}}_{k, l}$ analogous to the one for $\mathrm{Gr}_{k, l}$ in Subsection 1.4. Let $\xi_{k}^{\text {univ }} \rightarrow \mathrm{BU}(k)$ be the universal vector bundle with fiber $\mathbb{C}^{k}$. Then clearly $\mathbf{H}_{k, l}\left(\operatorname{End}\left(\xi_{k}^{\text {univ }}\right)\right) \simeq \widehat{\mathrm{Gr}}_{k, l}$, moreover under this identification the projection $\mathbf{H}_{k, l}\left(\operatorname{End}\left(\xi_{k}^{\text {univ }}\right)\right) \rightarrow \mathrm{BU}(k)$ coincides with the map $\widehat{\mathrm{Gr}}_{k, l} \rightarrow \mathrm{BU}(k)$ we have just defined by the commutative square.

Consider the following morphism of $\mathrm{U}(k)$-fibrations:

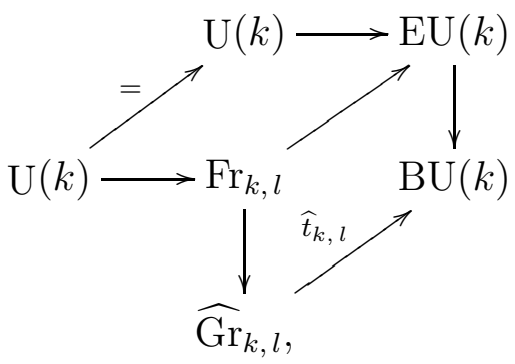

where $\widehat{t}_{k, l}$ is the classifying map for the canonical $\mathrm{U}(k)$-bundle over $\widehat{\mathrm{Gr}}_{k, l}$ and by $\mathrm{EU}(k)$ we denote the total space of the universal principal $\mathrm{U}(k)$-bundle which is contractible. A simple computation with homotopy sequences of the fibrations shows that $\widehat{t}_{k, l *}: \pi_{2 r}\left(\widehat{\operatorname{Gr}}_{k, l}\right) \rightarrow \pi_{2 r}(\mathrm{BU}(k)), r \leq \min \{k, l\}$ is just the monomorphism $\mathbb{Z} \rightarrow \mathbb{Z}, 1 \mapsto k \cdot 1$ (note that the odd-dimensional stable homotopy groups of both spaces are equal to 0$)$. This implies ([12], Theorem 2.1) that the direct limit map $\widehat{t_{k}}: \widehat{\mathrm{Gr}} \rightarrow \underset{r}{\lim } \mathrm{BU}\left(k^{r}\right)=: \mathrm{BU}\left(k^{\infty}\right)$ is just the localization away from $k$ (in the sense 
that $k$ is invertible; in particular, $\underset{r}{\lim } \mathrm{BU}\left(k^{r}\right)=\mathrm{BU}\left[\frac{1}{k}\right]$ is a $\mathbb{Z}\left[\frac{1}{k}\right]$-local space), where $\widehat{\mathrm{Gr}}:=\lim _{(k, l)=1} \widehat{\mathrm{Gr}}_{k, l}$.

The diagram (3) gives us the diagram

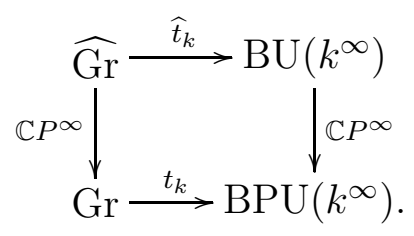

The left-hand vertical arrow in terms of bundles can be described as an assignment

$$
\left(\xi_{k^{n}},\left(\operatorname{End}\left(\xi_{k^{n}}\right), \mu, \widetilde{M}_{(k l)^{n}}\right)\right) \mapsto\left(\operatorname{End}\left(\xi_{k^{n}}\right), \mu, \widetilde{M}_{(k l)^{n}}\right)
$$

$\widehat{t}_{k}$ as an assignment

$$
\left(\xi_{k^{n}},\left(\operatorname{End}\left(\xi_{k^{n}}\right), \mu, \widetilde{M}_{(k l)^{n}}\right)\right) \mapsto \xi_{k^{n}}
$$

and also $t_{k}$ and the right-hand arrow as $\left(A_{k^{n}}, \mu, \widetilde{M}_{(k l)^{n}}\right) \mapsto A_{k^{n}}$ and $\xi_{k^{n}} \mapsto \operatorname{End}\left(\xi_{k^{n}}\right)$ respectively, where $\left(\operatorname{End}\left(\xi_{k^{n}}\right), \mu, \widetilde{M}_{(k l)^{n}}\right)$ and $\left(A_{k^{n}}, \mu, \widetilde{M}_{(k l)^{n}}\right)$ denote some FABs.

The space $\widehat{\mathrm{Gr}}$ is an $H$-space with respect to the multiplication induced by the tensor product of bundles. It can be proved that $\widehat{\mathrm{Gr}} \cong \mathrm{BU} \otimes$ as $H$-spaces. Let us also recall that $\mathrm{BU}_{\otimes} \cong \mathrm{BSU}_{\otimes} \times \mathbb{C} P^{\infty}$ and $\mathrm{Gr} \cong \mathrm{BSU}_{\otimes}$ as $H$-spaces, hence $\widehat{\mathrm{Gr}} \cong \mathrm{Gr} \times \mathbb{C} P^{\infty}$. In particular, the $H$-space $\widehat{\mathrm{Gr}}$ represents the functor of the "multiplicative group" of the ring $\widetilde{\mathrm{K}}_{\mathbb{C}}$, i.e. the functor $X \mapsto \widetilde{\mathrm{K}}_{\mathbb{C}}(X)$, where $\widetilde{\mathrm{K}}_{\mathbb{C}}(X)$ is considered as a group with respect to the operation $\xi * \eta=\xi+\eta+\xi \eta, \xi, \eta \in \widetilde{\mathrm{K}}_{\mathbb{C}}(X)$ (here $\widetilde{\mathrm{K}}_{\mathbb{C}}$ is the reduced complex $K$-functor).

$\operatorname{By} \widehat{\mathrm{AB}}^{1}(X), \widehat{\mathrm{AB}}^{k}(X)$ denote the groups $[X, \widehat{\mathrm{Gr}}]$ and $\left[X, \mathrm{BU}\left(k^{\infty}\right)\right]$ respectively. We also have the group homomorphism $\widehat{t}_{k *}(X): \widehat{\mathrm{AB}}^{1}(X) \rightarrow \widehat{\mathrm{AB}}^{k}(X)$ induced by $\widehat{t}_{k}$.

\section{Homotopy invariants related to algebra bundles}

\subsection{Reminder: The classical Brauer group}

Undoubtedly, the most important invariant constructed by means of matrix algebra bundles (called in this context "Azumaya bundles") is the Brauer group which plays an important role not only in Topology but also in Algebraic Geometry (where it turns out to be a birational invariant of varieties [7]). In this subsection we give a brief survey of the classical results concerning its "purely topological" version.

So let $X$ be a finite $C W$-complex. Consider the set of isomorphism classes of locally trivial bundles over $X$ with fiber $M_{k}(\mathbb{C})$ with an arbitrary integer $k>1$. On the set of such bundles consider the following equivalence relation:

$$
A_{k} \sim B_{l} \Leftrightarrow A_{k} \otimes \widetilde{M}_{m} \cong B_{l} \otimes \widetilde{M}_{n} \quad \text { for some } m, n>1
$$


where by $\widetilde{M}_{r}$ we denote a trivial bundle over $X$ with fiber $M_{r}(\mathbb{C})$. By $\left\{C_{m}\right\}$ denote the stable equivalence class of $C_{m}$. It can easily be checked that the product $\left\{A_{k}\right\} \circ\left\{B_{l}\right\}:=\left\{A_{k} \otimes B_{l}\right\}$ is well defined and equips the set of stable equivalence classes with the structure of Abelian group. We denote this group by $\widetilde{\mathrm{AB}}(X)$. Clearly, $\widetilde{\mathrm{AB}}(X)=\lim _{\vec{k}} \widetilde{\mathrm{AB}}^{k}(X)$, where the direct limit is taken over the natural homomorphisms $\widetilde{\mathrm{AB}}^{k}(X) \rightarrow \widetilde{\mathrm{AB}}^{k m}(X)$ induced by the assignments $A_{k^{n}} \mapsto A_{(k m)^{n}}:=A_{k^{n}} \otimes \widetilde{M}_{m^{n}}$.

Now consider the following more coarse stable equivalence relation:

$$
A_{k} \sim B_{l} \Leftrightarrow \exists \xi_{m}, \eta_{n} \text { such that } A_{k} \otimes \operatorname{End}\left(\xi_{m}\right) \cong B_{l} \otimes \operatorname{End}\left(\eta_{n}\right),
$$

where by $\xi_{m}, \eta_{n}$ we denote vector bundles over $X$ of rank $m, n$, respectively. By $\left[C_{m}\right]$ denote the stable equivalence class of $M_{m}(\mathbb{C})$-bundle $C_{m}$. The tensor product of bundles induces a group structure on the set of such stable equivalence classes. It is just the classical topological Brauer group $\operatorname{Br}(X)$.

Let us give a homotopic description of $\operatorname{Br}(X)$. Consider the fibration

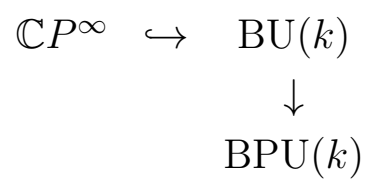

corresponding to the exact sequence of groups

$$
1 \rightarrow \mathrm{U}(1) \rightarrow \mathrm{U}(k) \rightarrow \mathrm{PU}(k) \rightarrow 1 .
$$

The first obstruction $\alpha(f)$ for the lifting of a map $f: X \rightarrow \operatorname{BPU}(k)$ in (7) belongs to $H^{3}(X ; \mathbb{Z})(\underline{6}$, Ch.5) and has order $k$ (the last assertion follows from the fact that $\alpha(f)=\delta(\beta(f))$, where $\beta(f) \in H^{2}(X ; \mathbb{Z} / k \mathbb{Z})$ is the first obstruction for the lifting in the bundle $\mathrm{B} \mu_{k} \hookrightarrow \mathrm{BSU}(k) \rightarrow \mathrm{BPU}(k)$ and $\delta: H^{2}(X ; \mathbb{Z} / k \mathbb{Z}) \rightarrow H^{3}(X ; \mathbb{Z})$ is the coboundary homomorphism).

Let us introduce the following notation for the direct limits:

$$
\mathrm{B} \mathcal{U}:=\lim _{\vec{k}} \mathrm{BU}(k), \quad \mathrm{B} \mathcal{P U}:=\lim _{\vec{k}} \operatorname{BPU}(k),
$$

where $k$ runs over all positive integers and the limits are taken over the maps inducing by the tensor product (in particular, BU is a $\mathbb{Q}$-space). Recall that

$$
\mathrm{B} \mathcal{U}=\prod_{q \geq 1} \mathrm{~K}(2 q, \mathbb{Q}), \quad \mathrm{B} \mathcal{P U}=\mathrm{K}(2, \mathbb{Q} / \mathbb{Z}) \times \prod_{q \geq 2} \mathrm{~K}(2 q, \mathbb{Q}), \quad \mathbb{C} P^{\infty}=\mathrm{K}(2, \mathbb{Z}),
$$

and we have the fibration

$$
\mathbb{C} P^{\infty} \hookrightarrow \mathrm{K}(2, \mathbb{Q}) \rightarrow \mathrm{K}(2, \mathbb{Q} / \mathbb{Z})
$$


corresponding to the exact sequence of the coefficients groups

$$
0 \rightarrow \mathbb{Z} \rightarrow \mathbb{Q} \rightarrow \mathbb{Q} / \mathbb{Z} \rightarrow 0
$$

We consider every space $\mathbb{C} P^{\infty}, \mathrm{B} \mathcal{U}$, and $\mathrm{B} \mathcal{P U}$ as an $H$-space with the multiplication induced by the tensor product of bundles which it classifies (for example, $\mathbb{C} P^{\infty}$ classifies complex line bundles, and there is the isomorphism $\mathbb{C} P^{\infty} \cong \mathrm{K}(2, \mathbb{Z}), \zeta \mapsto c_{1}(\zeta)$ of $H$ spaces, where $c_{1}$ is the first Chern class).

Thus, after taking the limit as $k \rightarrow \infty$ in (7) we get the fibration

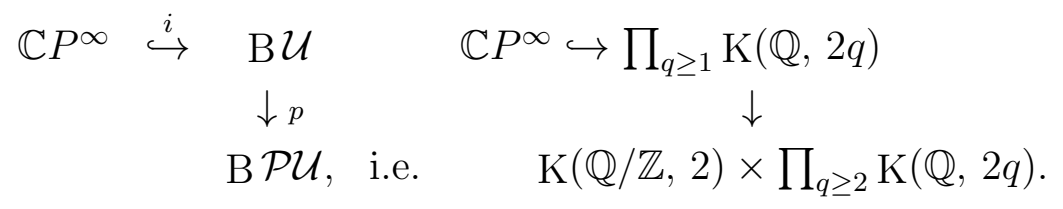

The $H$-space $\mathrm{B} \mathcal{P U}$ represents the homotopy functor $X \mapsto \widetilde{\mathrm{AB}}(X)$ on the category of finite $C W$-complexes. Indeed, since we take the direct limit $\mathrm{B} \mathcal{P U}=\underset{\vec{k}}{\lim \mathrm{BPU}}(k)$ over the maps of classifying spaces $\operatorname{BPU}(k) \stackrel{i_{k, m}}{\longrightarrow} \operatorname{BPU}(k m)$ such that $i_{k, m}^{*}\left(A_{k m}^{\text {univ }}\right)=$ $A_{k}^{\text {univ }} \otimes \widetilde{M}_{m}$ (where by $A_{r}^{\text {univ }}$ we denote the universal $M_{r}(\mathbb{C})$-bundle over $\operatorname{BPU}(r)$ ), the stable equivalence relation (6) appears.

Now it is easy to see that $\operatorname{Br}(X)=\operatorname{coker}\left\{p_{*}:[X, \mathrm{~B} \mathcal{U}] \rightarrow[X, \mathrm{~B} \mathcal{P U}]\right\}$, i.e. $\operatorname{Br}(X)=$ $\operatorname{coker}\{[X, \mathrm{~K}(\mathbb{Q}, 2)] \rightarrow[X, \mathrm{~K}(\mathbb{Q} / \mathbb{Z}, 2)]\}=\operatorname{coker}\left\{H^{2}(X ; \mathbb{Q}) \rightarrow H^{2}(X ; \mathbb{Q} / \mathbb{Z})\right\}$. Using the exact sequence of cohomology groups induced by the sequence of coefficients

$$
0 \rightarrow \mathbb{Z} \rightarrow \mathbb{Q} \rightarrow \mathbb{Q} / \mathbb{Z} \rightarrow 0
$$

we see that coker $\left\{H^{2}(X ; \mathbb{Q}) \rightarrow H^{2}(X ; \mathbb{Q} / \mathbb{Z})\right\}=\operatorname{im}\left\{H^{2}(X ; \mathbb{Q} / \mathbb{Z}) \stackrel{\delta}{\rightarrow} H^{3}(X ; \mathbb{Z})\right\}$ (here $\delta$ is the coboundary homomorphism), i.e. $\operatorname{Br}(X)=H_{\text {tors }}^{3}(X ; \mathbb{Z})[7$.

The explicit form of the isomorphism $\operatorname{Br}(X) \cong H_{\text {tors }}^{3}(X ; \mathbb{Z})$ can be described as follows. Recall that the structure group of a bundle $A_{k}$ with fiber $M_{k}(\mathbb{C})$ is $\operatorname{Aut}\left(M_{k}(\mathbb{C})\right)=\mathrm{PGL}_{k}(\mathbb{C})$ and $\mathrm{PGL}_{k}(\mathbb{C})$ contains $\mathrm{PU}(k)$ as a strong deformation retract. The obstruction theory asserts $([\underline{6}$, Ch.5) that the first (and unique!) obstruction for the lifting of $\mathrm{PU}(k)$-bundle to a $\mathrm{U}(k)$-bundle belongs to the group $H^{3}\left(X ; \pi_{2}\left(\mathbb{C} P^{\infty}\right)\right)$ (see fibration (7)). Therefore the assignment

$$
A_{k} \mapsto\{\text { the first obstruction for the lifting }\}
$$

gives us the required description of the isomorphism $\operatorname{Br}(X) \cong H_{\text {tors }}^{3}(X ; \mathbb{Z})$.

Thus, any element of the group $H_{\text {tors }}^{3}(X ; \mathbb{Z})$ can be realized as an obstruction for the lifting of some PU-bundle to a U-bundle (or equivalent a PGL-bundle to a GL-bundle). Remark 23. Let us remark that it is not necessarily that for $\alpha \in H^{3}(X ; \mathbb{Z})$ such that $k \alpha=0$ there exists a bundle $A_{k}$ with fiber $M_{k}(\mathbb{C})$ whose invariant is equal to $\alpha$ (see [2, p.11). 
Remark 24. Now suppose $A_{k}$ has a lifting $\xi_{k}$ (i.e. $\operatorname{End}\left(\xi_{k}\right)=A_{k}$ ). Then the obstruction theory says that $\xi_{k}$ is determined by $A_{k}$ up to taking the tensor product with a line bundle $\zeta$ over $X$. Indeed, it was already mentioned that $H^{2}\left(X ; \pi_{2}\left(\mathbb{C} P^{\infty}\right)\right)=H^{2}(X ; \mathbb{Z})$ is isomorphic to the group of line bundles with respect to the tensor product; from the other hand, for any line bundle $\zeta$ we have $\operatorname{End}\left(\xi_{k} \otimes \zeta\right)=\operatorname{End}\left(\xi_{k}\right)$.

Remark 25. Note that for any fixed integer $k>1$ one can develop the corresponding theory of bundles with fibers of the form $M_{k^{n}}(\mathbb{C})$ for arbitrary $n \in \mathbb{N}$. In this way one can define the $k$-primary component $\operatorname{Br}_{k}(X)$ of the Brauer group as $\operatorname{coker}\left\{p_{k *}:\left[X, \mathrm{BU}\left(k^{\infty}\right)\right] \rightarrow\left[X, \mathrm{BPU}\left(k^{\infty}\right)\right]\right\}$.

\subsection{An obstruction for an embedding of a locally trivial alge- bra bundle into a trivial one}

In this subsection using the $\operatorname{Hom}\left(M_{k}(\mathbb{C}), M_{k l}(\mathbb{C})\right)$-bundle $\mathbf{H}_{k, l}\left(A_{k}^{\text {univ }}\right) \rightarrow \operatorname{BPU}(k)$ (which has been studied in Subsection 1.4) we define topological obstructions for an embedding of a locally trivial matrix algebra bundle into a trivial one. Note that we consider only embeddings that are fiberwise homomorphisms of central algebras.

More precisely, let $A_{k}$ be an $M_{k}(\mathbb{C})$-bundle over a finite $C W$-complex $X$. In this subsection we construct a cohomological obstruction for the existence of an embedding $\mu: A_{k} \rightarrow X \times M_{k l}(\mathbb{C})$ such that $\mu_{x}\left(A_{k}\right)_{x} \subset M_{k l}(\mathbb{C})$ is a central subalgebra for any $x \in X$. This obstruction equals 0 iff $A_{k}$ is the core of some $\operatorname{FAB}\left(A_{k}, \mu, \widetilde{M}_{k l}\right)$ over $X$. Remark 26. Note that nontrivial obstructions can exist (after taking the limit as in Remark 30 below) only if $(k, l)=1$. In other words, all the obstructions vanish in the stable case if we reject this condition.

Consider the fibration

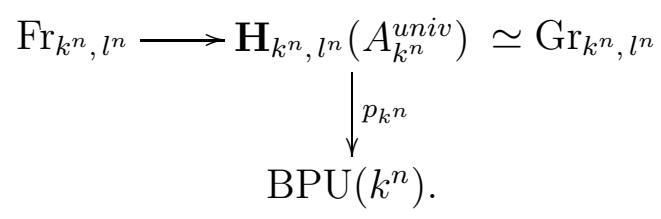

Let $X$ be a finite $C W$-complex, $\operatorname{dim}(X)<2 l^{n}$. Then for a given map $f: X \rightarrow \operatorname{BPU}\left(k^{n}\right)$ we have the first obstruction $\alpha(f) \in H^{2 i}\left(X ; \pi_{2 i-1}\left(\operatorname{Fr}_{k^{n}, l^{n}}\right)\right)$ for the lifting in (9]) ([6], Ch.5), where $\pi_{2 i-1}\left(\operatorname{Fr}_{k^{n}, l^{n}}\right)=\mathbb{Z} / k^{n} \mathbb{Z}$ and $\pi_{2 i}\left(\operatorname{Fr}_{k^{n}, l^{n}}\right)=0$ because $i<l^{n}$. But we have shown in Subsection 1.4 that a lifting of $f: X \rightarrow \operatorname{BPU}\left(k^{n}\right)$ in (9) is the same thing as an embedding $f^{*}\left(A_{k^{n}}^{\text {univ }}\right) \hookrightarrow X \times M_{(k l)^{n}}(\mathbb{C})$. Therefore the class $\alpha(f)$ is the first obstruction for the existence of an embedding of $f^{*}\left(A_{k^{n}}^{\text {univ }}\right)$ into the trivial bundle $X \times M_{(k l)^{n}}(\mathbb{C})($ recall that $(k, l)=1)$.

Note that for a mapping $f: X \rightarrow \mathrm{BU}\left(k^{n}\right)$ we can also define the obstruction for an embedding of the bundle $f^{*}\left(\operatorname{End}\left(\xi_{k^{n}}^{\text {univ }}\right)\right)=\operatorname{End}\left(f^{*}\left(\xi_{k^{n}}^{\text {univ }}\right)\right)$ into the trivial one $X \times$ 
$M_{(k l)^{n}}(\mathbb{C})$. For this purpose instead of bundle (9) we should consider the bundle

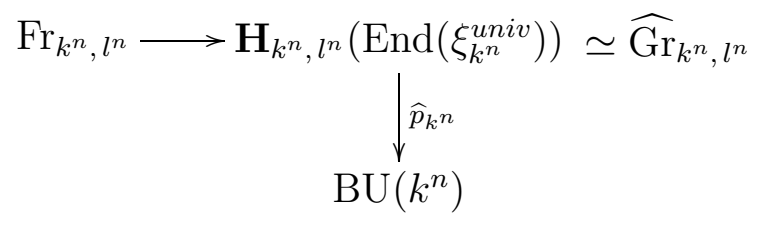

(see Remark 221). Clearly, the obstructions will be the same as in the "projective" case (in connection with this note that the map $\mathrm{BU}\left(k^{n}\right) \rightarrow \mathrm{BPU}\left(k^{n}\right)$ induces an isomorphism of cohomology groups $\left.H^{2}\left(\mathrm{BPU}\left(k^{n}\right) ; \mathbb{Z} / k^{n} \mathbb{Z}\right) \cong H^{2}\left(\mathrm{BU}\left(k^{n}\right) ; \mathbb{Z} / k^{n} \mathbb{Z}\right)\right)$.

Now take $k, l, m, n$ such that $(k m, l n)=1$. Notice that there is the natural map

$\phi_{k, l ; m, n}: \operatorname{Hom}\left(M_{k}(\mathbb{C}), M_{k l}(\mathbb{C})\right) \times \operatorname{Hom}\left(M_{m}(\mathbb{C}), M_{m n}(\mathbb{C})\right) \rightarrow \operatorname{Hom}\left(M_{k m}(\mathbb{C}), M_{k l m n}(\mathbb{C})\right)$

induced by the tensor product of matrix algebras.

Remark 27. If we identify $\operatorname{Hom}\left(M_{k}(\mathbb{C}), M_{k l}(\mathbb{C})\right)$ with $\operatorname{Fr}_{k, l}$ then the map $\phi_{k, l ; m, n}$ can be described as follows. One can easily verify that for a $k$-frame $\left\{\alpha_{i, j} \mid 1 \leq i, j \leq k\right\}$ in $M_{k l}(\mathbb{C})$ and an m-frame $\left\{\beta_{r, s} \mid 1 \leq r, s \leq m\right\}$ in $M_{m n}(\mathbb{C})$ the collection $\left\{\alpha_{i, j} \otimes \beta_{r, s} \mid\right.$ $1 \leq i, j \leq k, 1 \leq r, s \leq m\}$ is a $k m$-frame in $M_{k l m n}(\mathbb{C})=M_{k l}(\mathbb{C}) \otimes M_{m n}(\mathbb{C})$. Thus we have the natural map $\mathrm{Fr}_{k, l} \times \mathrm{Fr}_{m, n} \rightarrow \operatorname{Fr}_{k m, l n}$ which coincides with $\phi_{k, l ; m, n}$ under the mentioned identification.

Clearly, we also have the corresponding map of bundles

$$
\varphi_{k, l ; m, n}: \mathbf{H}_{k, l}\left(A_{k}^{\text {univ }}\right) \times \mathbf{H}_{m, n}\left(A_{m}^{\text {univ }}\right) \rightarrow \mathbf{H}_{k m, l n}\left(A_{k m}^{\text {univ }}\right)
$$

such that the diagram

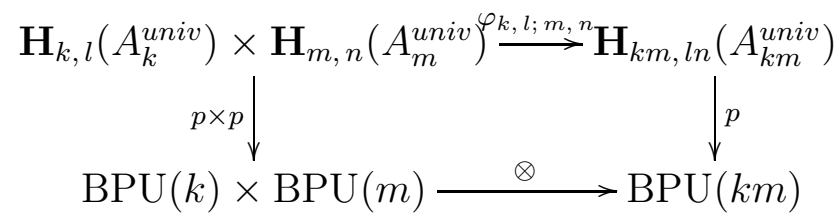

commutes. Obviously, the map $\varphi_{k, l ; m, n}$ can be identified with the natural map $\mathrm{Gr}_{k, l} \times \mathrm{Gr}_{m, n} \rightarrow \mathrm{Gr}_{k m, l n}$ of matrix Grassmannians induced by the tensor product of matrix algebras. These maps define the above described (see Subsection 2.2) $H$-space structure on $\mathrm{Gr}:=\lim _{(k, l)=1} \mathrm{Gr}_{k, l} \cong \mathrm{BSU}_{\otimes}$.

The same is true in the "unitary" case.

Lemma 28. The homotopy type of $\operatorname{Fr}_{k, l^{\infty}}$ does not depend on the choice of $l,(k, l)=1$.

Proof. Take $m$ such that $(k, m)=1$. Define the map $\alpha_{k, l, m}: \operatorname{Fr}_{k, l} \rightarrow \operatorname{Fr}_{k, l m}$ by the commutative diagram:

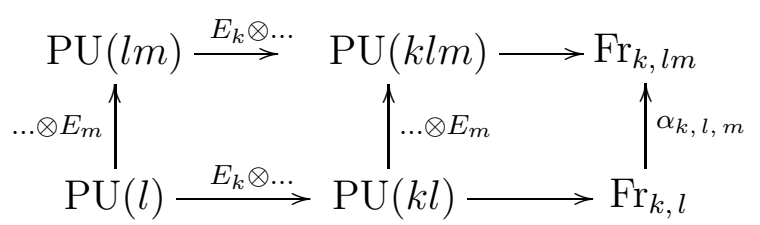


(the rows are fibrations). One can easily verify that the map $\alpha_{k, l, m}: \mathrm{Fr}_{k, l} \rightarrow \mathrm{Fr}_{k, l m}$ is a homotopy equivalence in dimensions $<2 l$. Now using the diagram

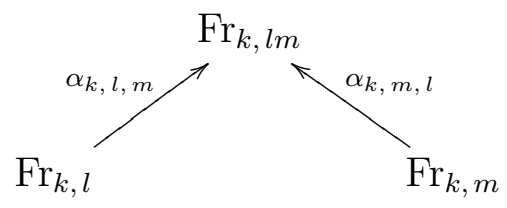

we get the desired assertion.

The proved lemma allows us to omit $l$ in the following notation.

Suppose $(m, n)=1, k|m, l| n(\Rightarrow(k, l)=1)$. Consider the following morphism of fiber bundles:

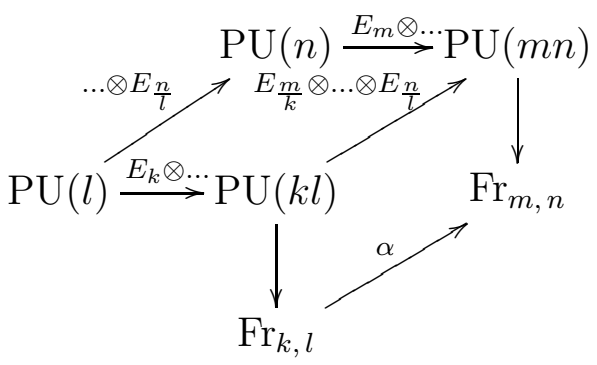

Using such maps $\alpha$ we can form the direct limit $\operatorname{Fr}_{k^{\infty}, l^{\infty}}$ whose homotopy type does not depend on the choice of $l$, so we shall denote it just by $\operatorname{Fr}_{k}$.

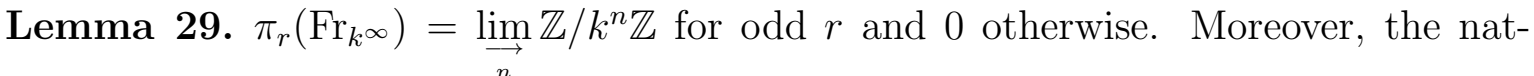
ural embedding $\operatorname{Fr}_{k^{n}} \hookrightarrow \operatorname{Fr}_{k^{\infty}}$ induces the monomorphisms $\pi_{2 r-1}\left(\operatorname{Fr}_{k^{n}}\right) \cong \mathbb{Z} / k^{n} \mathbb{Z} \hookrightarrow$ $\pi_{2 r-1}\left(\operatorname{Fr}_{k^{\infty}}\right), 1 \leq r \leq \infty$.

Proof follows from simple calculations with homotopy sequences of obvious fibrations.

So we can define the direct limit (as $n \rightarrow \infty)$ of (9)

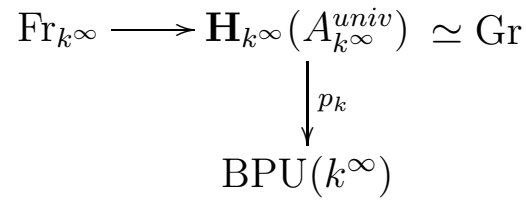

and of (10)

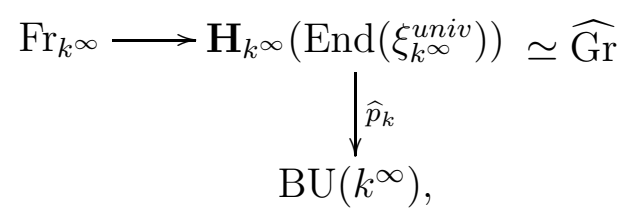

respectively. All the mappings are the homomorphisms of $H$-spaces. In particular, we see that $p_{k}, \widehat{p}_{k}$ are fiber substitutes for maps $t_{k}, \widehat{t}_{k}$ (see Section 2 ), respectively.

Remark 30. Suppose we are given a map $f: X \rightarrow \operatorname{BPU}\left(k^{n}\right)$ such that $\alpha(f) \neq 0, \alpha(f) \in$ $H^{2 i}\left(X ; \pi_{2 i-1}\left(\operatorname{Fr}_{k^{n}, l^{n}}\right)\right)$ (see fibration (91)), $\operatorname{dim} X<2 l^{n}$. Then the corresponding limit 
obstruction in (13) is also nontrivial. One can prove this using the following fact: for $(k m, \ln )=1$ the natural map $\mathrm{Fr}_{k, l} \rightarrow \mathrm{Fr}_{k m, l n}$ induces monomorphisms of the homotopy groups: $\mathbb{Z} / k \mathbb{Z} \cong \pi_{2 i-1}\left(\mathrm{Fr}_{k, l}\right) \hookrightarrow \pi_{2 i-1}\left(\mathrm{Fr}_{k m, l n}\right) \cong \mathbb{Z} / k m \mathbb{Z}, i \leq l$.

Remark 31. Let $\mathrm{F}_{k}(X)$ be the set of equivalence classes of $M_{k^{n}}\left(\mathbb{C}\right.$ )-bundles $A_{k^{n}}$ (for arbitrary $n \in \mathbb{N}$ ) with respect to the following equivalence relation:

$$
A_{k^{m}} \sim A_{k^{n}} \Leftrightarrow \text { there are FAB's cores } B_{k^{r}}, B_{k^{s}}
$$

such that $A_{k^{m}} \otimes B_{k^{r}} \cong A_{k^{n}} \otimes B_{k^{s}}(\Rightarrow m+r=n+s)$.

Obviously, $\mathrm{F}_{k}(X)$ is an Abelian group with respect to the operation induced by the tensor product of bundles. Moreover, $\mathrm{F}_{k}(X)=\operatorname{coker}\left\{p_{k *}:[X, \mathrm{Gr}] \rightarrow\left[X, \operatorname{BPU}\left(k^{\infty}\right)\right]\right\}$ $\left(=\operatorname{im}\left\{h_{k *}:\left[X, \operatorname{BPU}\left(k^{\infty}\right)\right] \rightarrow\left[X, \mathrm{BN}_{k^{\infty}}^{\times}\right]\right\}\right.$, see the next subsection). Note that the first cohomological obstruction for the lifting in (13) is well-defined on such equivalence classes.

Although the group $\mathrm{F}_{k}(X)$ looks like the Brauer group $\operatorname{Br}_{k}(X)$, the actual analog of the latter group will be defined in the next subsection.

\subsection{A generalized Brauer group}

Consider exact sequence (14) of $H$-spaces. Our goal is to extend it to the right. So at the next step we have to define a "classifying space" for $\mathrm{Fr}_{k^{\infty}}$. One possible approach uses the fact that $\mathrm{Fr}_{k^{\infty}}$ is an infinite loop space (as a fiber of the localization map $\widehat{t}_{k}: \mathrm{BU} U_{\otimes}=\widehat{\mathrm{Gr}} \rightarrow \mathrm{BU}\left[\frac{1}{k}\right]_{\otimes}=\mathrm{BU}\left(k^{\infty}\right)$ for $\mathrm{BU}_{\otimes} ; \mathrm{BU}_{\otimes}$ is an infinite loop space due to G.B. Segal [1]). In this way one obtains the fibration

$$
\widehat{\mathrm{Gr}} \stackrel{\widehat{t}_{k}}{\longrightarrow} \mathrm{BU}\left(k^{\infty}\right) \stackrel{\widehat{h}_{k}}{\longrightarrow} \mathrm{BFr}_{k^{\infty}}
$$

where $\mathrm{BFr}_{k^{\infty}}$ is the base of the universal principal $\mathrm{Fr}_{k^{\infty}}$-fibration, $\Omega\left(\mathrm{BFr}_{k^{\infty}}\right)=\mathrm{Fr}_{k^{\infty}}$.

But we present a more direct approach by replacing the loop spaces by groups of the same homotopy type. More precisely, we introduce topological groups $\mathrm{GL}_{k^{\infty}}(\mathcal{K}(H)), \mathrm{N}_{k^{\infty}}^{\times}, \mathrm{GL}_{1}\left(\Delta_{k^{\infty}}\right)^{0}=\mathrm{GL}_{1}(\mathcal{C}(H))^{0}$ together with homomorphisms $\mathrm{GL}_{k^{\infty}}(\mathcal{K}(H)) \rightarrow \mathrm{N}_{k^{\infty}}^{\times}, \mathrm{N}_{k^{\infty}}^{\times} \rightarrow \mathrm{GL}_{1}\left(\Delta_{k^{\infty}}\right)^{0}$ forming the exact sequence of groups

$$
\mathrm{GL}_{k^{\infty}}(\mathcal{K}(H)) \rightarrow \mathrm{N}_{k^{\infty}}^{\times} \rightarrow \mathrm{GL}_{1}\left(\Delta_{k^{\infty}}\right)^{0}
$$

which is homotopy equivalent to the fibration

$$
\mathrm{U}\left(k^{\infty}\right) \rightarrow \mathrm{Fr}_{k} \rightarrow \widehat{\mathrm{Gr}}
$$

(see diagram (44)). Then we identify $\mathrm{BFr}_{k^{\infty}}$ with the classifying space $\mathrm{BN}_{k^{\infty}}$ and the map $\widehat{h}_{k}: \mathrm{BU}\left(k^{\infty}\right) \rightarrow \operatorname{BFr}_{k^{\infty}}$ with the map of classifying spaces $\operatorname{BGL}_{k}(\mathcal{K}(H)) \rightarrow$ 
$\mathrm{BN}_{k^{\infty}}^{\times}$, induced by the above group homomorphism. The main advantage of this approach is that any element of the defined below generalized Brauer group can be represented by a locally trivial bundle with the structure group $\mathrm{N}_{k}^{\times}$(for some $k \in \mathbb{N}$ ).

So let $H$ be a separable Hilbert space, $\mathcal{B}(H)$ and $\mathcal{K}(H)$ the algebra of bounded operators in $\operatorname{End}(H)$ and the ideal of compact operators in $\mathcal{B}(H)$, respectively. By $\mathcal{C}(H)$ denote the Calkin algebra $\mathcal{B}(H) / \mathcal{K}(H)$. Put

$$
\Delta_{k}:=\left\{\left(\begin{array}{cccc}
\lambda & 0 & \ldots & 0 \\
0 & \lambda & \ldots & 0 \\
\vdots & \vdots & \ddots & \vdots \\
0 & 0 & \ldots & \lambda
\end{array}\right) \mid \lambda \in \mathcal{C}(H)\right\} \subset M_{k}(\mathcal{C}(H)) .
$$

Clearly, $\Delta_{k}$ is a subalgebra in $M_{k}(\mathcal{C}(H))$ isomorphic to $\mathcal{C}(H)$. Let $\pi_{k}: M_{k}(\mathcal{B}(H)) \rightarrow$ $M_{k}(\mathcal{C}(H))$ be the natural epimorphism. Let $\mathrm{N}_{k}$ be the subalgebra $\pi_{k}^{-1}\left(\Delta_{k}\right) \subset$ $M_{k}(\mathcal{B}(H)), \mathrm{N}_{k}^{\times}:=\mathrm{GL}_{1}\left(\mathrm{~N}_{k}\right)=\mathrm{N}_{k} \cap \mathrm{GL}_{k}(\mathcal{B}(H))$ its multiplicative group. It is a closed subgroup (in the norm topology) in $\mathrm{GL}_{k}(\mathcal{B}(H))$. The groups $\mathrm{N}_{k^{n}}^{\times}$play a crucial role in the further constructions. In particular, we shall show that in contrast to the group $\mathrm{GL}_{1}\left(\mathcal{B}(H)\right.$ ), they are not contractible, if $n>0$ (note that $\mathrm{N}_{k^{0}}^{\times} \cong \mathrm{GL}_{1}(\mathcal{B}(H))$ ).

Remark 32. One can replace $\mathrm{N}_{k}^{\times}$by the homotopy equivalent (polar decomposition!) unitary group $\mathrm{N}_{k}^{\times} \cap \mathrm{U}_{k}(\mathcal{B}(H))$, where $\mathrm{U}_{k}(\mathcal{B}(H)) \subset M_{k}(\mathcal{B}(H))$ is the group of unitary operators contained in $M_{k}(\mathcal{B}(H))$.

Remark 33. Let us remark that there is an obvious way to transfer our definition of $\mathrm{N}_{k}^{\times}$to the algebraic $K$-theory of a ring $R$ (using the analogy between $\mathcal{C}(H)$ and $\Sigma R$ ).

The following results about Calkin algebra are needed for the sequel. Let $\pi: \mathcal{B}(H) \rightarrow \mathcal{C}(H)$ be the canonical epimorphism. By $\mathrm{GL}_{1}(\mathcal{C}(H))^{0}$ denote the connected component of the unit in $\mathrm{GL}_{1}(\mathcal{C}(H))$. In other words, it is the image of the space $\operatorname{Fred}(H)^{0}$ of zero index Fredholm operators under the map $\left.\pi\right|_{\operatorname{Fred}(H)}: \operatorname{Fred}(H) \rightarrow$ $\mathrm{GL}_{1}(\mathcal{C}(H)$ ) (which is a fibration with fiber an affine space over $\mathcal{K}(H)$ ). Let $\bar{\pi}: \mathrm{GL}_{1}(\mathcal{B}(H)) \rightarrow \mathrm{GL}_{1}(\mathcal{C}(H))^{0}$ be the birestriction of $\pi$ to the multiplicative subgroups. Clearly, $\operatorname{ker}(\bar{\pi})=\mathrm{GL}_{1}(\mathcal{K}(H))$, where $\mathrm{GL}_{1}(\mathcal{K}(H))$ is the group of invertible operators of the form $1+K, K \in \mathcal{K}(H)$. It is known [9] that this group is homotopy equivalent to the infinite unitary group $\mathrm{U}:=\underset{\longrightarrow}{\longrightarrow} \mathrm{U}(n)$ (with respect to the standard inclusions). Recall also that the group $\mathrm{GL}_{1}(\mathcal{C}(H))\left(\mathrm{GL}_{1}(\mathcal{C}(H))^{0}\right)$ is homotopy equivalent to $\mathbb{Z} \times \mathrm{BU}$ (BU respectively). Finally note that $\mathrm{GL}_{k}(\mathcal{K}(H)):=$ \{the group of invertible $k \times k$-matrices of the form $\left.1+K \mid K \in M_{k}(\mathcal{K}(H))\right\}$ is isomorphic to $\mathrm{GL}_{1}(\mathcal{K}(H))$.

Consider the group epimorphisms $\bar{\pi}_{k}: \mathrm{GL}_{k}(\mathcal{B}(H)) \rightarrow \mathrm{GL}_{k}(\mathcal{C}(H))^{0}$ and $\widetilde{\pi}_{k}: \mathrm{N}_{k}^{\times} \rightarrow$ $\mathrm{GL}_{1}\left(\Delta_{k}\right)^{0}$ induced by $\pi_{k}$. Clearly their kernels coincide with the subgroup $\mathrm{GL}_{k}(\mathcal{K}(H))$. 
Lemma 34. The topological group $\mathrm{N}_{k}^{\times}$has the following homotopy groups: $\pi_{2 r}\left(\mathrm{~N}_{k}^{\times}\right)=$ $0, \pi_{2 r-1}\left(\mathrm{~N}_{k}^{\times}\right)=\mathbb{Z} / k \mathbb{Z}, r \geq 1$.

Proof easily follows from the commutative diagram of exact sequences

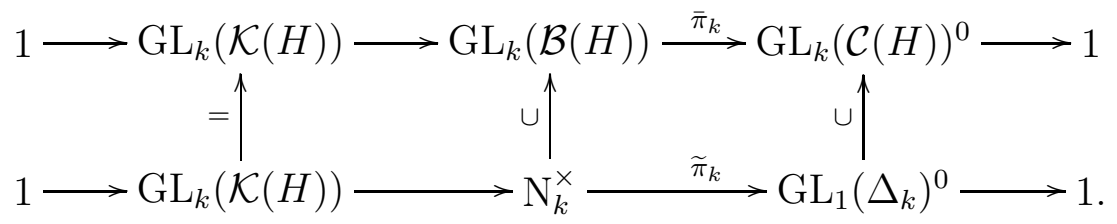

Indeed, recall that the group $\mathrm{GL}_{k}(\mathcal{B}(H)) \cong \mathrm{GL}_{1}(\mathcal{B}(H))$ is contractible (the polar decomposition + Kuiper's theorem). In particular, upper row is just the path fibration. Now the required assertion follows from the nontrivial piece of the corresponding homotopy sequences:

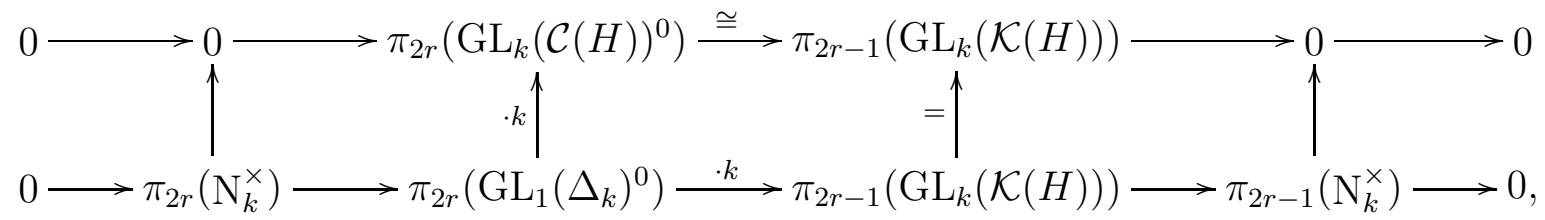

where $\cdot k$ means the homomorphism which takes the group generator 1 to $k \cdot 1$.

Remark 35. Since the inclusion $\mathrm{GL}_{1}\left(\Delta_{k}\right)^{0} \hookrightarrow \mathrm{GL}_{k}(\mathcal{C}(H))^{0}$ in diagram (16) is a classifying map for the lower row (considered as a $\mathrm{GL}_{k}(\mathcal{K}(H))$-fibration), we have the fibration

$$
\mathrm{N}_{k}^{\times} \stackrel{\tilde{\pi}_{k}}{\longrightarrow} \mathrm{GL}_{1}\left(\Delta_{k}\right)^{0} \longrightarrow \mathrm{GL}_{k}(\mathcal{C}(H))^{0} .
$$

Because of $\Omega\left(\mathrm{GL}_{1}\left(\Delta_{k}\right)^{0}\right) \simeq \mathrm{GL}_{1}(\mathcal{K}(H)), \Omega\left(\mathrm{GL}_{k}(\mathcal{C}(H))^{0}\right) \simeq \mathrm{GL}_{k}(\mathcal{K}(H))$, we also obtain the fibration

$$
\mathrm{GL}_{1}(\mathcal{K}(H)) \longrightarrow \mathrm{GL}_{k}(\mathcal{K}(H)) \longrightarrow \mathrm{N}_{k}^{\times}
$$

where the inclusion $\mathrm{GL}_{1}(\mathcal{K}(H)) \rightarrow \mathrm{GL}_{k}(\mathcal{K}(H))$ is the following:

$$
\alpha \mapsto\left(\begin{array}{cccc}
\alpha & 0 & \ldots & 0 \\
0 & \alpha & \ldots & 0 \\
\vdots & \vdots & \ddots & \vdots \\
0 & 0 & \ldots & \alpha
\end{array}\right)
$$

$\left(k \times k\right.$-matrix); in particular, $\mathrm{N}_{k}^{\times}$is homotopy equivalent to the homogeneous space $\mathrm{GL}_{k}(\mathcal{K}(H)) / \mathrm{GL}_{1}(\mathcal{K}(H))$.

Remark 36. Since $\mathrm{BGL}_{1}(\mathcal{K}(H)) \simeq \mathrm{GL}_{1}\left(\Delta_{k}\right)^{0}$ we see that there is the map $\mathrm{N}_{k}^{\times} \rightarrow$ $\operatorname{BGL}_{1}(\mathcal{K}(H))$ which is equivalent to the epimorphism $\tilde{\pi}_{k}: \mathrm{N}_{k}^{\times} \rightarrow \mathrm{GL}_{1}\left(\Delta_{k}\right)^{0}, \operatorname{ker}\left(\widetilde{\pi}_{k}\right)=$ $\mathrm{GL}_{k}(\mathcal{K}(H))$; analogously, there is the map $\mathrm{BGL}_{1}(\mathcal{K}(H)) \rightarrow \mathrm{BGL}_{k}(\mathcal{K}(H))$ which is equivalent to the natural inclusion $\mathrm{GL}_{1}\left(\Delta_{k}\right)^{0} \rightarrow \mathrm{GL}_{k}(\mathcal{C}(H))^{0}$, where $\mathcal{C}(H)$ is the Calkin algebra as above. 
Consider the homomorphisms $\mathrm{U}(k n) \stackrel{\kappa_{n}}{\hookrightarrow} \mathrm{U}(k(n+1))$,

$$
\left(\begin{array}{cccc}
\alpha_{11} & \alpha_{12} & \ldots & \alpha_{1 k} \\
\alpha_{21} & \alpha_{22} & \ldots & \alpha_{2 k} \\
\vdots & \vdots & \ddots & \vdots \\
\alpha_{k 1} & \alpha_{k 2} & \ldots & \alpha_{k k}
\end{array}\right) \mapsto\left(\begin{array}{ccccccc}
\alpha_{11} & 0 & \alpha_{12} & 0 & \ldots & \alpha_{1 k} & 0 \\
0 & 1 & 0 & 0 & \ldots & 0 & 0 \\
\alpha_{21} & 0 & \alpha_{22} & 0 & \ldots & \alpha_{2 k} & 0 \\
0 & 0 & 0 & 1 & \ldots & 0 & 0 \\
\vdots & \vdots & \vdots & \vdots & \ddots & \vdots & \vdots \\
\alpha_{k 1} & 0 & \alpha_{k 2} & 0 & \ldots & \alpha_{k k} & 0 \\
0 & 0 & 0 & 0 & \ldots & 0 & 1
\end{array}\right)
$$

where $\alpha_{i j} \in M_{n}(\mathbb{C})$. Let $i_{n}: \mathrm{U}(n) \hookrightarrow \mathrm{U}(n+1)$ be the standard inclusion

$$
\alpha \mapsto\left(\begin{array}{ll}
\alpha & 0 \\
0 & 1
\end{array}\right), \alpha \in \mathrm{U}(n) .
$$

Consider also the homomorphisms $\mathrm{U}(n) \stackrel{E_{k} \otimes \cdots}{\longrightarrow} \mathrm{U}(k n)$. One can easily check that $E_{k} \otimes$ $\left(i_{n} \alpha\right)=\kappa_{n}\left(E_{k} \otimes \alpha\right) \forall \alpha \in \mathrm{U}(n)$. Thus, we obtain the well-defined map of the direct limits:

$$
\tau_{k}: \mathrm{U}=\lim _{\overrightarrow{i_{n}}} \mathrm{U}(n) \rightarrow \lim _{\overrightarrow{\kappa_{n}}} \mathrm{U}(k n) \cong \mathrm{U}
$$

induced by the tensor product with $E_{k}$ (cf. diagram (18)).

Lemma 37. The space $\mathrm{Fr}_{k}$ is the fiber of the map $\mathrm{B} \tau_{k}$ : BU $\rightarrow \mathrm{BU}$ induced by the group homomorphism (19).

Proof. Obviously, the map $\mathrm{BU}\left(l^{n}\right) \stackrel{[k] \otimes \ldots}{\longrightarrow} \mathrm{BU}\left(k l^{n}\right)$ induced by the tensor product with a trivial bundle of rank $k$ is equivalent to the map of these classifying spaces induced by the group homomorphism $\mathrm{U}\left(l^{n}\right) \stackrel{E_{k} \otimes_{\ldots}}{\longrightarrow} \mathrm{U}\left(k l^{n}\right)$. Hence $\operatorname{Fr}_{k, l^{n}}$ is the fiber of $\mathrm{BU}\left(l^{n}\right) \stackrel{[k] \otimes \ldots}{\longrightarrow}$ $\mathrm{BU}\left(k l^{n}\right)$. It easily follows from the commutative diagram (cf. diagram (11))

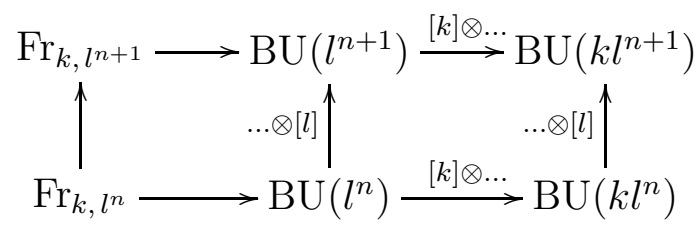

that the natural inclusions $\mathrm{Fr}_{k, l^{n}} \rightarrow \mathrm{Fr}_{k, l^{n+1}}$ are weak homotopy equivalences ( $\Rightarrow$ homotopy equivalences because $\operatorname{Fr}_{k, l}$ is a $C W$-complex) up to dimension $\sim 2 l^{n}$ (recall that $(k, l)=1)$.

The following proposition makes the statement of Lemma 34 more precise (cf. Lemma 29]. 
Proposition 38. There is a homotopy equivalence $\mathrm{Fr}_{k} \simeq \mathrm{N}_{k}^{\times}$such that the diagram (whose rows are the above fibrations (cf. Remarks 35 and 36) and the vertical arrows are homotopy equivalences)

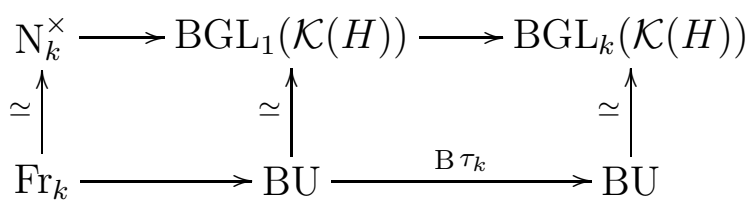

commutes up to homotopy.

Proof. We have the group homomorphism $\mathrm{U} \rightarrow \mathrm{GL}_{1}(\mathcal{K}(H))$ which is a homotopy equivalence. Clearly, this equivalence identifies the group homomorphisms $\tau_{k}: \mathrm{U} \rightarrow \mathrm{U}$ and $\mathrm{GL}_{1}(\mathcal{K}(H)) \rightarrow \mathrm{GL}_{k}(\mathcal{K}(H))$ (see (18) ). There is also the homotopy equivalence $\mathrm{BU} \rightarrow \mathrm{BGL}_{1}(\mathcal{K}(H))$ of classifying spaces and the corresponding maps of classifying spaces $\mathrm{B} \tau_{k}: \mathrm{BU} \rightarrow \mathrm{BU}$ and $\mathrm{BGL}_{1}(\mathcal{K}(H)) \rightarrow \mathrm{BGL}_{k}(\mathcal{K}(H))$ can also be identified. Therefore their homotopy fibers $\mathrm{Fr}_{k}$ and $\mathrm{N}_{k}^{\times}$are homotopy equivalent too, and the diagram is commutative.

Remark 39. Let us remark that the fibration (see (17))

$$
\mathrm{BGL}_{1}(\mathcal{K}(H)) \rightarrow \mathrm{BGL}_{k}(\mathcal{K}(H)) \rightarrow \mathrm{BN}_{k}^{\times}
$$

has the following interpretation. Let $\mathrm{EN}_{k}^{\times} \rightarrow \mathrm{BN}_{k}^{\times}$be the universal principal $\mathrm{N}_{k}^{\times}$bundle. Using the action of the subgroup $\mathrm{GL}_{k}(\mathcal{K}(H)) \subset \mathrm{N}_{k}^{\times}$on its fibers $\cong \mathrm{N}_{k}^{\times}$, we obtain (20) (because $\mathrm{BGL}_{1}(\mathcal{K}(H)) \cong \mathrm{GL}_{1}\left(\Delta_{k}\right)^{0} \cong \mathrm{N}_{k}^{\times} / \mathrm{GL}_{k}(\mathcal{K}(H))$ ).

Now consider the commutative diagram of group homomorphisms:

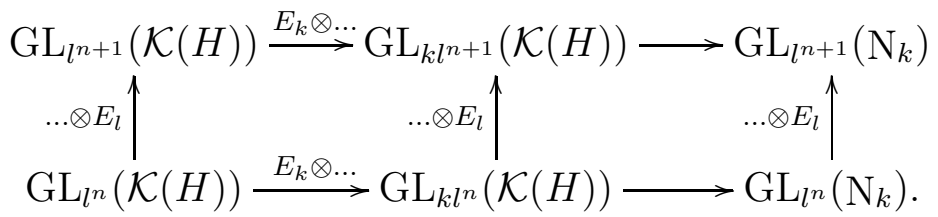

Since $(k, l)=1$, we see that the arrow $\operatorname{GL}_{l^{n}}\left(\mathrm{~N}_{k}\right) \rightarrow \mathrm{GL}_{l^{n+1}}\left(\mathrm{~N}_{k}\right)$ is a homotopy equivalence (cf. the proof of Lemma 37). Put $\operatorname{GL}_{l \infty}(\mathcal{K}(H)):=$ $\lim _{\vec{n}} \mathrm{GL}_{l^{n}}(\mathcal{K}(H)), \quad \mathrm{GL}_{k l^{\infty}}(\mathcal{K}(H)):=\underset{\vec{n}}{\lim _{\vec{n}}} \mathrm{GL}_{k l^{n}}(\mathcal{K}(H))$, where the direct limits are taken over the group homomorphisms $\mathrm{GL}_{l^{n}}(\mathcal{K}(H)) \stackrel{\cdots \otimes E_{l}}{\longrightarrow} \mathrm{GL}_{l^{n+1}}(\mathcal{K}(H))$ and $\mathrm{GL}_{k l^{n}}(\mathcal{K}(H)) \stackrel{\cdots \otimes E_{l}}{\longrightarrow} \mathrm{GL}_{k l^{n+1}}(\mathcal{K}(H))\left(\mathrm{GL}_{l \infty}\left(\mathrm{N}_{k}\right) \simeq \mathrm{GL}_{1}\left(\mathrm{~N}_{k}\right)=\mathrm{N}_{k}^{\times}\right)$. Thus, we have the fibration

$$
\mathrm{GL}_{l \infty}(\mathcal{K}(H)) \rightarrow \mathrm{GL}_{k l \infty}(\mathcal{K}(H)) \rightarrow \mathrm{N}_{k}^{\times}
$$

and hence also the fibration

$$
\mathrm{N}_{k}^{\times} \rightarrow \mathrm{BGL}_{l \infty}(\mathcal{K}(H)) \stackrel{[k] \otimes \ldots}{\longrightarrow} \mathrm{BGL}_{k l^{\infty}}(\mathcal{K}(H)) .
$$

Taking into account the obtained results, the proof of the following lemma is trivial. 
Lemma 40. There is the commutative (up to homotopy) diagram

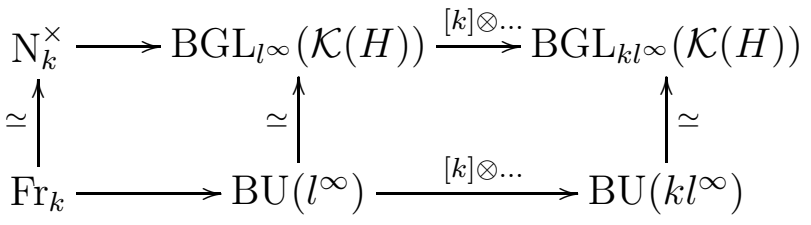

whose rows are fibrations and vertical arrows are homotopy equivalences.

Lemma 41. There is the commutative (up to homotopy) diagram

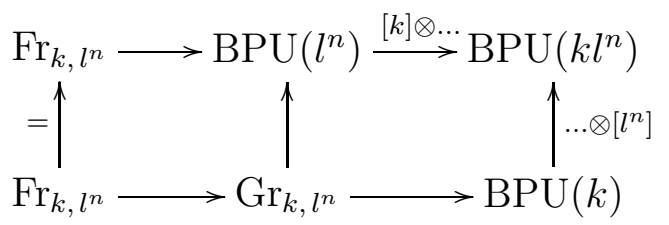

whose rows are the above fibrations.

Proof. Consider the mapping

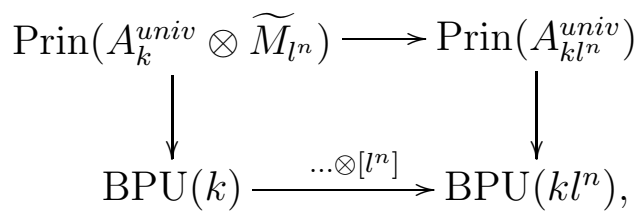

of principal $\mathrm{PU}\left(k l^{n}\right)$-bundles (so it is $\mathrm{PU}\left(k l^{n}\right)$-equivariant). We have the free action of the subgroup $E_{k} \otimes \mathrm{PU}\left(l^{n}\right) \subset \mathrm{PU}\left(k l^{n}\right)$ on their total spaces. Note that $\operatorname{Prin}\left(A_{k}^{\text {univ }} \otimes \widetilde{M}_{l^{n}}\right)$ is the fiber of the map $\operatorname{BPU}(k) \stackrel{\cdots \otimes\left[l^{n}\right]}{\longrightarrow} \operatorname{BPU}\left(k l^{n}\right)$, hence

$$
\operatorname{Prin}\left(A_{k}^{u n i v} \otimes \widetilde{M}_{l^{n}}\right) \cong \mathrm{PU}\left(k l^{n}\right) /\left(\mathrm{PU}(k) \otimes E_{l^{n}}\right) .
$$

Therefore its factor by $E_{k} \otimes \mathrm{PU}\left(l^{n}\right)$ is $\mathrm{Gr}_{k, l^{n}}$ and we obtain the required commutative diagram.

Replacing BPU by BU and Gr by $\widehat{\mathrm{Gr}}$ one obtains the analogous diagram in the unitary case.

Put $\widehat{\operatorname{Gr}}_{k}:=\underset{n}{\lim } \widehat{\operatorname{Gr}}_{k, l^{n}}$. We claim that this homotopy type does not depend on the choice of $l,(k, l)=1$. Indeed, the maps $\alpha_{k, l, m}$ and $\alpha_{k, m, l}$ in diagram (12) are $\mathrm{PU}(k)$-equivariant.

Corollary 42. There is the commutative (up to homotopy) diagram

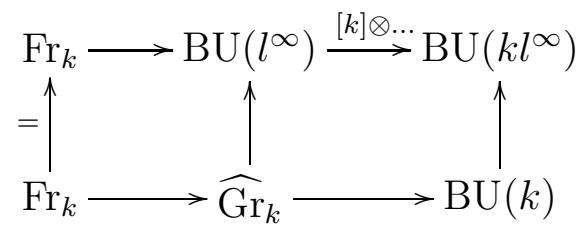

whose rows are the fibrations. 
Combining the previous corollary with Lemma 40, we obtain the following result.

Theorem 43. There is the fibration

$$
\widehat{\mathrm{Gr}}_{k} \rightarrow \mathrm{BU}(k) \rightarrow \mathrm{BN}_{k}^{\times}
$$

which actually is an extension of $\mathrm{Fr}_{k} \rightarrow \widehat{\mathrm{Gr}}_{k} \rightarrow \mathrm{BU}(k)$ to the right.

Proof. The upper fibration in diagram (21) can be extended to the right up to fibration

$$
\mathrm{BGL}_{l \infty}(\mathcal{K}(H)) \rightarrow \mathrm{BGL}_{k l^{\infty}}(\mathcal{K}(H)) \rightarrow \mathrm{BN}_{k}^{\times}
$$

The map $\mathrm{BU}(k) \rightarrow \mathrm{BN}_{k}^{\times}$is just the composition $\mathrm{BU}(k) \rightarrow \operatorname{BU}\left(k l^{\infty}\right) \simeq$ $\mathrm{BGL}_{k l^{\infty}}(\mathcal{K}(H)) \rightarrow \mathrm{BN}_{k}^{\times}$. It follows from the previous corollary that the homotopic fiber of this map is just $\widehat{\mathrm{Gr}}_{k}$.

Remark 44. Let us note an analogy between fibrations (17) and (22) (cf. the definition of generalized Brauer group below).

There is a continuous action of the group $\mathrm{N}_{k}^{\times}$on different spaces; for example one can consider the action on the algebra $\mathrm{N}_{k}$ by inner automorphisms. So one can think of a bundle with the structure group $\mathrm{N}_{k}^{\times}$as a locally-trivial $\mathrm{N}_{k}$-bundle.

Let $\mathbf{F}_{k}$ be the functor on bundles, induced by the above map of classifying spaces $\mathrm{BU}(k) \rightarrow \mathrm{BN}_{k}^{\times}$. By analysis of the previous constructions, one can obtain the explicit form of the corresponding group homomorphism $\mathrm{U}(k) \rightarrow \mathrm{N}_{k}^{\times}$. For example (for $k=2$ ) it is as follows:

$$
\left(\begin{array}{cc}
\alpha & \beta \\
\gamma & \delta
\end{array}\right) \mapsto\left(\begin{array}{cccccccc}
\alpha & 0 & 0 & \ldots & \beta & 0 & 0 & \ldots \\
0 & 1 & 0 & \ldots & 0 & 0 & 0 & \ldots \\
0 & 0 & 1 & \ldots & 0 & 0 & 0 & \ldots \\
\vdots & \vdots & \vdots & \ddots & \vdots & \vdots & \vdots & \ddots \\
\gamma & 0 & 0 & \ldots & \delta & 0 & 0 & \ldots \\
0 & 0 & 0 & \ldots & 0 & 1 & 0 & \ldots \\
0 & 0 & 0 & \ldots & 0 & 0 & 1 & \ldots \\
\vdots & \vdots & \vdots & \ddots & \vdots & \vdots & \vdots & \ddots
\end{array}\right)
$$

Corollary 45. Suppose we are given an $\mathbb{C}^{k}$-bundle $\xi_{k}$ over a finite $C W$-complex $X$. Then $\mathbf{F}_{k}\left(\xi_{k}\right)$ is a trivial bundle iff $\operatorname{End}\left(\xi_{k}\right)$ is the core (see Subsection 2.3) of some $F A B$ over $X$ (i.e. iff there exists an embedding $\operatorname{End}\left(\xi_{k}\right) \hookrightarrow X \times M_{k m}(\mathbb{C})$ for some sufficiently large $m,(k, m)=1)$.

Proof. Since (22) is a fibration, we have the corresponding exact sequence of pointed sets $\left[X, \widehat{\mathrm{Gr}}_{k}\right] \rightarrow[X, \mathrm{BU}(k)] \rightarrow\left[X, \mathrm{BN}_{k}^{\times}\right]$. Now the required assertion follows from the discussion after diagram (5). 
Remark 46. Recall that we have already interpreted the lifting problem in the fibration $\operatorname{Fr}_{k} \rightarrow \widehat{\operatorname{Gr}}_{k} \simeq \mathbf{H}_{k}\left(\operatorname{End}\left(\xi_{k}^{u n i v}\right)\right) \rightarrow \mathrm{BU}(k)$ in terms of the existence of an embedding into a trivial bundle, see the discussion around diagram (10).

Remark 47. Let us return to the fibration (22). Since $\widehat{\operatorname{Gr}}_{k}=\operatorname{Gr}_{k} \underset{\mathrm{BPU}(k)}{\times} \mathrm{BU}(k)$ (see Subsection 2.6) it seems very reliable that the above map $\mathrm{BU}(k) \rightarrow \mathrm{BN}_{k}^{\times}$factors through $\mathrm{BPU}(k)$ i.e. there exists a map $\mathrm{BPU}(k) \rightarrow \mathrm{BN}_{k}^{\times}$(with fiber $\mathrm{Gr}_{k}$ ) such that the diagram

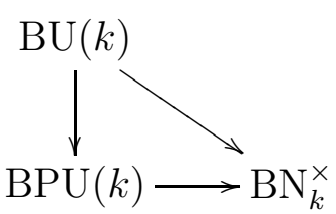

commutes. This might show that the generalized Brauer group actually is a generalization of the classical Brauer group (cf. diagram (24)).

Remark 48. It is interesting to apply the obtained results to the equivalence relation, considered in Remark 31. More precisely, we see that (in notation of the remark) $A_{k^{m}} \sim A_{k^{n}} \Leftrightarrow \mathbf{F}_{k^{m}}\left(A_{k^{m}}\right)$ and $\mathbf{F}_{k^{n}}\left(A_{k^{n}}\right)$ are "stable isomorphic".

It is obvious that the Kronecker product with the unit $k \times k$-matrix $E_{k}$ induces the group homomorphisms $\mathrm{N}_{k^{0}}^{\times} \rightarrow \mathrm{N}_{k}^{\times} \rightarrow \mathrm{N}_{k^{2}}^{\times} \rightarrow \ldots$. For the topological group $\mathrm{N}_{k^{n}}^{\times}$we have its classifying space $\mathrm{BN}_{k^{n}}^{\times}$. Since $\mathrm{B}$ is a functor, we see that the above homomorphisms induce the corresponding maps of classifying spaces $\mathrm{BN}_{k^{0}}^{\times} \rightarrow \mathrm{BN}_{k}^{\times} \rightarrow$ $\mathrm{BN}_{k^{2}}^{\times} \rightarrow \ldots$

Consider the homomorphisms $\mathrm{GL}_{1}(\mathcal{K}(H)) \rightarrow \mathrm{GL}_{k}(\mathcal{K}(H)) \rightarrow \mathrm{GL}_{k^{2}}(\mathcal{K}(H)) \rightarrow \ldots$ induced by the Kronecker product with the unit $k \times k$-matrix. Their direct limit is the localization map $\mathrm{GL}_{1}(\mathcal{K}(H)) \rightarrow \mathrm{GL}_{k^{\infty}}(\mathcal{K}(H))$.

Corollary 49. There is the diagram

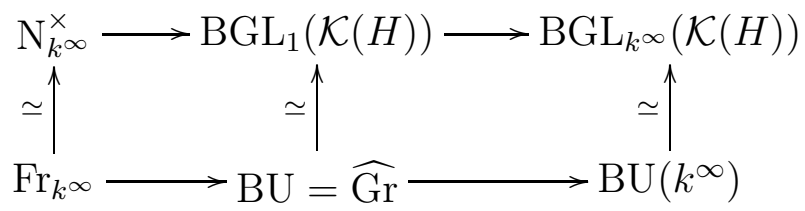

which is commutative up to homotopy.

Proof follows from Proposition 38 .

Thus, instead of fibration (15) we can consider the homotopy equivalent fibration

$$
\mathrm{BGL}_{1}(\mathcal{K}(H)) \stackrel{\widehat{t}_{k}^{\prime}}{\longrightarrow} \mathrm{BGL}_{k} \infty(\mathcal{K}(H)) \stackrel{\widehat{h}_{k}^{\prime}}{\longrightarrow} \mathrm{BN}_{k^{\infty}}^{\times}
$$

(which is the direct limit of (20) ). 
Now we want to define a structure of homotopy commutative $H$-space on $\mathrm{BN}_{k^{\infty}}^{\times}$ which turn the above fibration into an exact sequence of $H$-spaces (i.e.

$$
\left[X, \mathrm{BGL}_{1}(\mathcal{K}(H))\right] \stackrel{\widehat{t}_{k *}^{\prime}}{\longrightarrow}\left[X, \mathrm{BGL}_{k^{\infty}}(\mathcal{K}(H))\right] \stackrel{\widehat{h}_{k *}^{\prime}}{\longrightarrow}\left[X, \mathrm{BN}_{k^{\infty}}^{\times}\right]
$$

is an exact sequence of Abelian groups for any finite $C W$-complex $X$ ). Unfortunately, we do not know any direct way to do this in terms of $\mathrm{N}_{k^{n}}^{\times}$-bundles.

Remark 50. For example, there are mappings $\mathrm{N}_{k^{m}}^{\times} \times \mathrm{N}_{k^{n}}^{\times} \rightarrow \mathrm{N}_{k^{m+n}}^{\times}$defined by the Kronecker product of matrices $M_{k^{m}}(\mathcal{B}(H)) \times M_{k^{n}}(\mathcal{B}(H)) \rightarrow M_{k^{m+n}}(\mathcal{B}(H))$ (cf. Remark 27). Indeed, one can easily verify (using the fact that $\mathcal{K}(H)$ is a two-sided ideal in $\mathcal{B}(H)$ ) that for any $A \in \mathrm{N}_{k^{m}}^{\times}, B \in \mathrm{N}_{k^{n}}^{\times}$their Kronecker product $A \otimes B$ satisfies the condition $\bar{\pi}_{k^{m+n}}(A \otimes B) \in \mathrm{GL}_{1}\left(\Delta_{k^{m+n}}\right)^{0} \subset \mathrm{GL}_{k^{m+n}}(\mathcal{C}(H))^{0}$, i.e. $A \otimes B \in \mathrm{N}_{k^{m+n}}^{\times}$. But these mappings are not homomorphisms, so they do not define the corresponding mappings of classifying spaces.

So we have to return to fibration (15). Let $\mathfrak{b} \mathfrak{u}_{\otimes}^{i}$ be the generalized cohomology theory defined by the infinite loop space $\mathrm{BU}_{\otimes}$, with the zero term $\mathfrak{b} \mathfrak{u}_{\otimes}^{0}(X)=\left[X, \mathrm{BU}_{\otimes}\right][1]$. Then fibration (15) corresponds to the exact sequence of Abelian groups $\mathfrak{b} \mathfrak{u}_{\otimes}^{0}(X) \rightarrow$ $\mathfrak{b} \mathfrak{u}_{\otimes}^{0}\left(X, \mathbb{Z}\left[\frac{1}{k}\right]\right) \rightarrow \mathfrak{b} \mathfrak{u}_{\otimes}^{0}\left(X ; \mathbb{Z}\left[\frac{1}{k}\right] / \mathbb{Z}\right)$ associated with the exact sequence of groups of coefficients $0 \rightarrow \mathbb{Z} \rightarrow \mathbb{Z}\left[\frac{1}{k}\right] \rightarrow \mathbb{Z}\left[\frac{1}{k}\right] / \mathbb{Z} \rightarrow 0$ (note that $\mathbb{Z}\left[\frac{1}{k}\right] / \mathbb{Z}=\underset{n}{\lim } \mathbb{Z} / k^{n} \mathbb{Z}$ ).

Since $\mathfrak{b} \mathfrak{u}_{\otimes}^{0}\left(X ; \mathbb{Z}\left[\frac{1}{k}\right] / \mathbb{Z}\right)=\left[X, \mathrm{BN}_{k^{\infty}}^{\times}\right]$is an Abelian group, we see that the space $\mathrm{BN}_{k^{\infty}}^{\times}$is equipped with the $H$-space structure with the required property. Put $\mathrm{FB}^{k}(X):=\left[X, \mathrm{BN}_{k^{\infty}}^{\times}\right]$. Recall that the groups $[X, \widehat{\mathrm{Gr}}]$ and $\left[X, \mathrm{BU}\left(k^{\infty}\right)\right]$ we denoted by $\widehat{\mathrm{AB}}^{1}(X)$ and $\widehat{\mathrm{AB}}^{k}(X)$, respectively. We also defined the natural transformation (indeed the localization away from $k$ ) $\widehat{t}_{k *}: \widehat{\mathrm{AB}}^{1} \rightarrow \widehat{\mathrm{AB}}^{k}$ induced by $\widehat{t}_{k}$. Using the previous arguments we obtain the exact sequence of Abelian groups

$$
\widehat{\mathrm{AB}}^{1}(X) \stackrel{\widehat{t}_{k *}(X)}{\longrightarrow} \widehat{\mathrm{AB}}^{k}(X) \stackrel{\widehat{h}_{k *}(X)}{\longrightarrow} \mathrm{FB}^{k}(X)
$$

for any finite $C W$-complex $X$.

Definition 51. For a finite $C W$-complex $X$ the $k$-primary component (cf. Remark 25) of the generalized Brauer group $\operatorname{GBr}_{k}(X)$ is the cokernel coker $\left\{\widehat{h}_{k *}(X): \widehat{\mathrm{AB}}^{k}(X) \rightarrow\right.$ $\left.\mathrm{FB}^{k}(X)\right\}$.

Now we want to explain why this definition is a natural generalization of the classical Brauer group. First of all, let us stress an analogy between the fibration

$$
\mathbb{C} P^{\infty} \rightarrow \mathrm{BU}\left(k^{\infty}\right) \rightarrow \mathrm{BPU}\left(k^{\infty}\right)
$$

(which relates to the definition of the classical group) and fibration (23) (or (15)). For example, one can verify that the homotopy sequence of the first fibration in dimension 
2 coincides with the homotopy sequence of the second fibration in all even dimensions. Therefore in some sense we get a two-periodic generalization of the classical Brauer group. The role of the Picard group (i.e. the group of isomorphism classes of line bundles with respect to the tensor product) in our case plays the group of bundles of the form $\left(\xi_{k},\left(\operatorname{End}\left(\xi_{k}\right), \mu, \widetilde{M}_{k l}\right)\right),(k, l)=1$ (see Subsection 2.6) with respect to the tensor product (cf. Remark 24).

Now it is suitable to consider direct limits (induced by the Kronecker product) over all natural numbers (not only over powers of a fixed $k$ ). In this way we get spaces

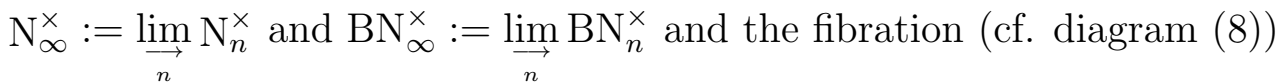

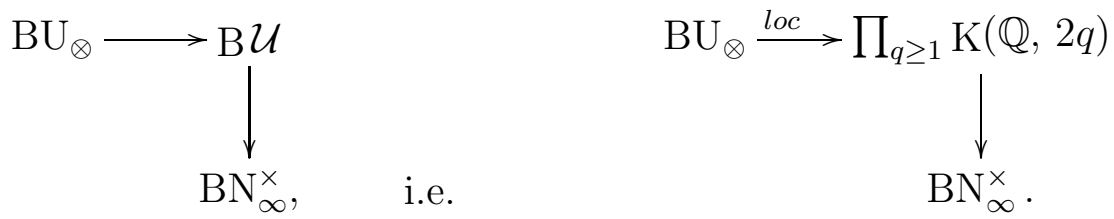

Since $\mathrm{BU}_{\otimes} \cong \mathbb{C} P^{\infty} \times \mathrm{BSU}_{\otimes}$, we see that this diagram splits:

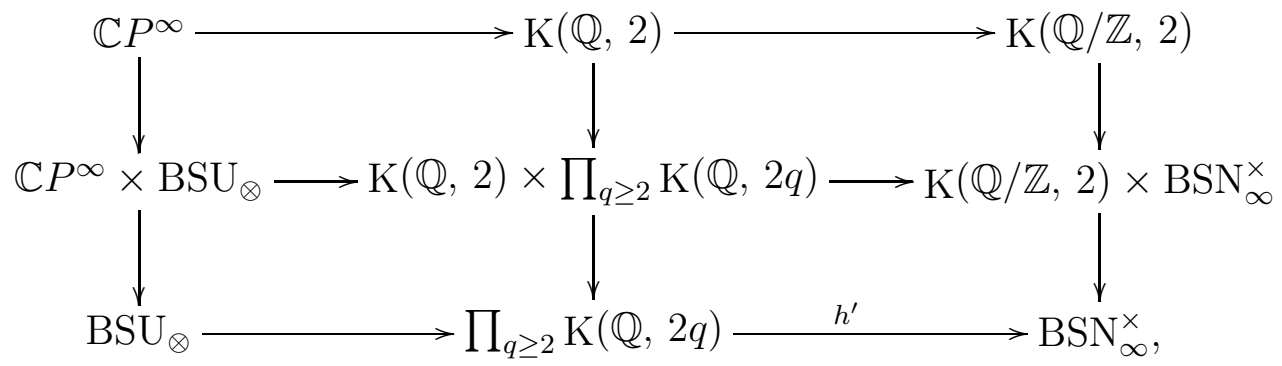

where $\mathrm{BSN}_{\infty}^{\times}$is defined by the diagram, $\mathrm{BN}_{\infty}^{\times}=\mathrm{K}(\mathbb{Q} / \mathbb{Z}, 2) \times \mathrm{BSN}_{\infty}^{\times}$. Notice that the upper fibration correspond to the classical Brauer group, so the generalized Brauer group is the product of the classical one with the group defined by the lower fibration.

Since $\mathrm{B} \mathcal{P U} \simeq \mathrm{K}(\mathbb{Q} / \mathbb{Z}, 2) \times \prod_{q \geq 2} \mathrm{~K}(\mathbb{Q}, 2 q)$, we obtain the map $h: \mathrm{B} \mathcal{P U} \rightarrow$ $\mathrm{BN}_{\infty}^{\times}, h=\mathrm{id}_{\mathrm{K}(\mathbb{Q} / \mathbb{Z}, 2)} \times h^{\prime}$ (with the homotopy fiber $\mathrm{Gr}=\mathrm{BSU}_{\otimes}$ ), where $h^{\prime}$ is defined in the previous diagram, such that the diagram

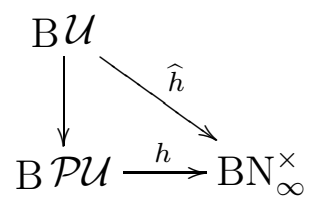

commutes. Put $\overline{\mathrm{GBr}}(X):=\operatorname{coker}\left\{h_{*}(X): \widetilde{\mathrm{AB}}(X) \rightarrow \mathrm{FB}(X)\right\}$ (where $\mathrm{FB}(X):=$ $\left.\left[X, \mathrm{BN}_{\infty}^{\times}\right]\right)$. Then we have the exact sequence of Abelian groups

$$
0 \rightarrow \mathrm{Br}(X) \rightarrow \operatorname{GBr}(X) \rightarrow \overline{\mathrm{GBr}}(X) \rightarrow 0,
$$

where the subgroup $\operatorname{Br}(X) \subset \operatorname{GBr}(X)$ corresponds to those $\mathrm{N}_{k}^{\times}$-bundles over $X$ whose structure group can be reduced to $\mathrm{PU}(k)$ (for sufficiently large $k$ ).

In other words, compared to the classical case, a whole new step is added to the procedure of the lifting. At first, we have to lift an $\mathrm{N}_{k}^{\times}$-bundle to a $\mathrm{PU}(k)$-bundle. The second step essentially coincides with the classical case. 


\subsection{Application: the twisted $K$-theory}

One of the possible application of the generalized Brauer group is to the twisted $K$ theory. More precisely, M. Atiyah and G. Segal posed in [2] the problem of finding of a geometrical approach to more general twistings than the one comes from projective unitary group $\mathrm{PU}(H)$ of the Hilbert space $H$. Recall that $\mathrm{PU}(H) \simeq \mathbb{C} P^{\infty}$, so $\operatorname{BPU}(H) \simeq \mathrm{K}(\mathbb{Z}, 3)$. For any element $\alpha \in H^{3}(X ; \mathbb{Z})=[X, \mathrm{~K}(\mathbb{Z}, 3)]$ (i.e. actually for a $\mathbb{C} P^{\infty}$-bundle with the structure group $\mathrm{PU}(H)$ ), in particular, for elements of $\operatorname{Br}(X)=H_{\text {tors }}^{3}(X ; \mathbb{Z})$, one can define a "twisted $K$-group" $K_{\alpha}^{0}(X)$ as the group of homotopy classes of sections of the $\operatorname{Fred}(H)$-bundle associated with $\alpha[2$. So let us give the analogous construction for bundles with the structure group $\mathrm{N}_{k}^{\times}$.

Remark 52. Recall that $\mathrm{N}_{k}^{\times}$-bundles represent elements of the generalized Brauer group. It seems reliable that the twisted $K$-theory associated with such a bundle depends only on the corresponding element of the generalized Brauer group, i.e. on the class of such a bundle modulo im $\left(\widehat{h}_{*}\right)$ (cf. Proposition 54 below).

Of course, the group $\mathrm{N}_{k}^{\times} \subset M_{k}(\mathcal{B}(H))$ naturally acts on $H^{k}$, but it is more fruitful to treat it as an extension of $\mathrm{N}_{1}^{\times}=\mathrm{N}_{k^{0}}^{\times}=\mathcal{B}(H)$. More precisely, put Fred $k_{k}:=$ $\mathrm{N}_{k} \cap \operatorname{Fred}\left(H^{k}\right)=\pi_{k}^{-1}\left(\mathrm{GL}_{1}\left(\Delta_{k}\right)\right)$, where $\operatorname{Fred}\left(H^{k}\right) \subset M_{k}(\mathcal{B}(H))$ denotes the space of Fredholm operators contained in $M_{k}(\mathcal{B}(H))$. We have the fibration Fred $_{k} \rightarrow \mathrm{GL}_{1}\left(\Delta_{k}\right)$ with fiber an affine space over $\operatorname{ker}\left(\pi_{k}\right)=M_{k}(\mathcal{K}(H))$. In particular, the projection Fred $_{k} \rightarrow \mathrm{GL}_{1}\left(\Delta_{k}\right)$ is a homotopy equivalence. Moreover, there are natural inclusions Fred $_{k} \rightarrow$ Fred $_{k^{2}} \rightarrow \ldots$ such that the diagram (consisting of homotopy equivalences)

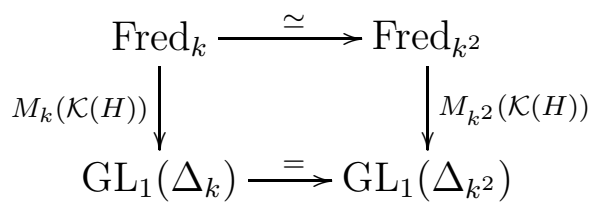

commutes.

Note also that there is the commutative diagram

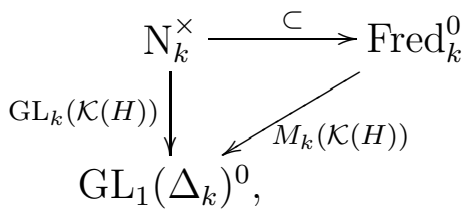

where both down-directed arrows are bundles with the marked fibres.

Clearly, the group $\mathrm{N}_{k}^{\times}$naturally acts on $\operatorname{Fred}_{k} \subset M_{k}(\mathcal{B}(H))$ by conjugations. Moreover, this action is compatible with the fibration $p_{k}: \operatorname{Fred}_{k} \rightarrow \mathrm{GL}_{1}\left(\Delta_{k}\right)$ in the sense that the diagram

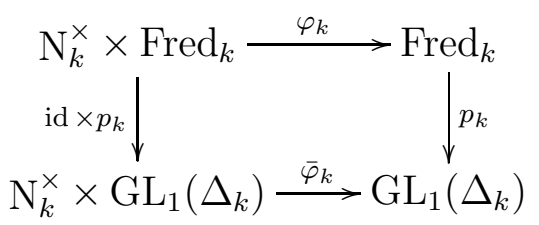


commutes (where $\varphi_{k}$ is the mentioned action and $\bar{\varphi}_{k}$ is the natural action on the group of invertible elements of Calkin algebra). In particular, the projection $p_{k}$ is $\mathrm{N}_{k}^{\times}$-equivariant, i.e. it commutes with the actions.

Thus for a given $\mathrm{N}_{k}^{\times}$-cocycle on $X$ we can associate the corresponding Fred $_{k}$-bundle $\widetilde{\text { Fred }}_{k} \rightarrow X$ and define the corresponding twisted $K$-theory as the set of homotopy classes of sections of this bundle. Since the $\operatorname{space}$ Fred $_{k}$ is a topological monoid with respect to the composition of Fredholm operators, and the group action preserves the composition, we see that this set is actually a group.

Remark 53. Note that the action $\mathrm{N}_{k}^{\times} \times \operatorname{Fred}\left(H^{k}\right) \rightarrow \operatorname{Fred}\left(H^{k}\right)$ can be extended to the action of the group $\mathrm{GL}_{k}(\mathcal{B}(H)) \supset \mathrm{N}_{k}^{\times}$which is contractible. Therefore for any Fred $\left(H^{k}\right)$-bundle with the structure group $\mathrm{N}_{k}^{\times}$there exists a fiberwise homeomorphism with the product bundle $X \times \operatorname{Fred}\left(H^{k}\right)$. But the action $\varphi_{k}: \mathrm{N}_{k}^{\times} \times \operatorname{Fred}_{k} \rightarrow \operatorname{Fred}_{k}$ cannot be extended to the action of $\mathrm{GL}_{k}(\mathcal{B}(H)$ ) (the latter group does not preserve the subspace $\left.\operatorname{Fred}_{k} \subset \operatorname{Fred}\left(H^{k}\right)\right)$. That's why we consider the action $\varphi_{k}$ on $\operatorname{Fred}_{k}$.

Proposition 54. Suppose our $\mathrm{N}_{k}^{\times}$-cocycle over $X$ comes from some $\mathrm{GL}_{k}(\mathcal{K}(H))$ cocycle (recall that $\mathrm{GL}_{k}(\mathcal{K}(H))$ is a subgroup in $\mathrm{N}_{k}^{\times}$). Then the corresponding twisted $K$-theory is isomorphic to the untwisted one $K_{\mathbb{C}}(X)$.

Proof. Recall that $\mathrm{GL}_{k}(\mathcal{K}(H)) \subset \mathrm{N}_{k}^{\times}$is the kernel of the natural homomorphism $\mathrm{N}_{k}^{\times} \rightarrow \mathrm{GL}_{1}\left(\Delta_{k}\right)^{0}$. The $\mathrm{N}_{k}^{\times}$-equivariant projection $p_{k}: \operatorname{Fred}_{k} \rightarrow \mathrm{GL}_{1}\left(\Delta_{k}\right)$ defines the fiberwise map

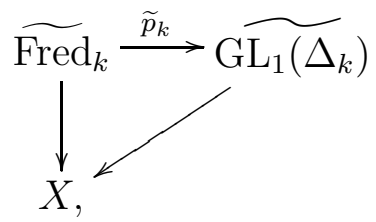

where $\left.\widehat{\mathrm{GL}_{1}\left(\Delta_{k}\right.}\right) \rightarrow X$ is the $\mathrm{GL}_{1}\left(\Delta_{k}\right)$-bundle associated with the same $\mathrm{N}_{k}^{\times}$-cocycle as $\widetilde{\text { Fred }}_{k}$ (using the action $\bar{\varphi}_{k}$, see diagram (25)). Since the subgroup $\mathrm{GL}_{k}(\mathcal{K}(H)) \subset \mathrm{N}_{k}^{\times}$ is contained in the kernel of the action $\bar{\varphi}_{k}: \mathrm{N}_{k}^{\times} \times \mathrm{GL}_{1}\left(\Delta_{k}\right) \rightarrow \mathrm{GL}_{1}\left(\Delta_{k}\right)$, we see that $\widehat{\mathrm{GL}_{1}\left(\Delta_{k}\right)} \rightarrow X$ is a trivial fibration. But the sets (actually groups) of homotopy classes of sections of two fibrations $\widetilde{\text { Fred }}_{k} \rightarrow X$ and $\widetilde{\mathrm{GL}_{1}\left(\Delta_{k}\right)} \rightarrow X$ are naturally isomorphic, because $\widetilde{p}_{k}$ is obviously a fiberwise homotopy equivalence.

Remark 55. Let us remark that our generalized Brauer group generalizes the classical Brauer group (corresponding to the elements of finite order in $H^{3}(X ; \mathbb{Z})$ ), but not the infinite order case corresponding to the Dixmier-Douady class of a principal PU $(H)$ bundle [8]. In order to obtain a further generalization we have to extend sequence (14) up to the sequence $\mathrm{BGL}_{k}(\mathcal{K}(H)) \stackrel{\widehat{h}_{k}^{\prime}}{\longrightarrow} \mathrm{BN}_{k^{\infty}}^{\times} \stackrel{\mathrm{B} \widetilde{\pi}_{k} \infty}{\longrightarrow} \mathrm{BGL}_{1}\left(\Delta_{k^{\infty}}\right)^{0}$ (cf. the fibration $\left.\mathrm{BU}\left(k^{\infty}\right) \rightarrow \mathrm{BPU}\left(k^{\infty}\right) \rightarrow \mathrm{K}(\mathbb{Z}, 3)\right)$. 


\section{References}

[1] J.F. Adams: Infinite Loop Spaces. Princeton, New Jersey, 1978.

[2] M. Atiyah, G. Segal: Twisted K-theory. arXiv preprint, math.KT/0407054.

[3] A.V. Ershov: Homotopy theory of bundles with fiber matrix algebra. Journal of Mathematical Sciences (New York) Vol.123, No.4 (2004), pp. 4198 - 4220.

[4] A.V. Ershov: Formal group laws over Hopf algebras and their application to complex cobordism theory. Preprint 39 (2002), Max-Planck-Institut für Mathematik.

[5] A.V. Ershov: Symmetries in complex cobordism theory related to stable equivalence classes of SU-bundles. Preprint 70 (2002), Max-Planck-Institut für Mathematik.

[6] Ph.A. Griffiths, J.W. Morgan: Rational Homotopy Theory and Differential Forms. Birkhäuser, 1981.

[7] A. Grothendieck: Le groupe de Brauer I. Sem. Bourbaki 1964/65, no. 290, $21 \mathrm{p}$.

[8] V. Mathai, R.B. Melrose, I.M. Singer: The index of projective families of elliptic operators. Geometry \& Topology Vol.9 (2005), 341-373.

[9] R.S. Palais: On the homotopy of certain groups of operators. Topology 3 (1965), 271-279.

[10] R.S. Pierce: Associative Algebras. Springer Verlag, 1982.

[11] G.B. Segal: Categories and cohomology theories. Topology 13 (1974), 293-312.

[12] D. Sullivan: Geometric Topology. MIT, Cambridge, Massachusetts, 1970.

e-mail: ershov@higeom.math.msu.su 